\title{
Les sites ecclésiaux et monastiques de l'archipel du Kvarner (Croatie), campagne 2017 : Martinšćica (île de Cres)
}

Morana Čaušević-Bully, Sébastien Bully, Petra Urbanová, Pascale Chevalier et Vivien Prigent

\section{(2) OpenEdition \\ Journals}

Édition électronique

URL : http://journals.openedition.org/cefr/2205

DOI : $10.4000 /$ cefr.2205

ISSN : 2282-5703

Éditeur

École française de Rome

Référence électronique

Morana Čaušević-Bully, Sébastien Bully, Petra Urbanová, Pascale Chevalier et Vivien Prigent, « Les sites ecclésiaux et monastiques de l'archipel du Kvarner (Croatie), campagne 2017 : Martinšćica (île de Cres) », Chronique des activités archéologiques de l'École française de Rome [En ligne], Balkans, mis en ligne le 11 décembre 2018, consulté le 23 mars 2020. URL : http://journals.openedition.org/cefr/2205 ; DOI : https://doi.org/10.4000/cefr.2205

Ce document a été généré automatiquement le 23 mars 2020

(c) École française de Rome 


\title{
Les sites ecclésiaux et monastiques de l'archipel du Kvarner (Croatie), campagne 2017 : Martinšćica (île de Cres)
}

\author{
Morana Čaušević-Bully, Sébastien Bully, Petra Urbanová, Pascale Chevalier \\ et Vivien Prigent
}

\section{NOTE DE L'AUTEUR}

L'équipe archéologique de Martinšćica était formée par :

Sébastien Bully et Morana Čaušević-Bully (direction), Georgie Baudry (étudiant en master d'archéologie, université Bourgogne Franche-Comté), Maxime Bolard (étudiant en master d'archéologie, UBFC), Thomas Chenal (archéologue-topographe, membre associé UMR 6298 ARTEHIS), Valentin Chevassu (doctorant-allocataire en archéologie, UBFC), Jessy Crochat (archéologue), Anaïs Deliste (étudiante en master d'anthropologie, université d'Aix-Marseille), Lucija Dugorepec (étudiante en master d'archéologie, université de Zagreb), Adrien Saggese (archéologue-céramologue, membre associé UMR 6298 ARTEHIS) et Ivan Valent (archéologue), avec les contributions de Petra Urbanová (UMR IRAMAT-Bordeaux), Vivien Prigent (UMR Orient et Méditerrannée) ; pour l'étude du verre, de Bernard Gratuze (archéomètre CNRS, IRAMAT-CEB Orléans) et Inès Pactat (doctorante-allocataire en archéologie, UBFC) ; Miro Vuković (archéologue-topographe, département de l'archéologie, université de Zagreb, couverture photographique par drone).

La troisième campagne de fouille programmée du site de Martinšćica (île de Cres) s'est déroulée entre le 29 mai et le 18 juin $^{1}$. L'opération archéologique a porté plus particulièrement sur la chapelle (Martinšćica 1bis-Esp. II) adossée contre le flanc sud de la grande église paléochrétienne, sur le bras sud du transept (Esp. Id) et sur une nouvelle annexe latérale (Esp. VIIIb) le long de la chapelle sud (fig. 1). L'opération a été 
prolongée entre les 11 et 16 septembre par une première tranche de travaux de conservation des maçonneries en élévation de l'église ${ }^{2}$ (fig. 2).

Fig. 1 - Vue générale par drone du site à l'achèvement de la fouille en juillet 2017.

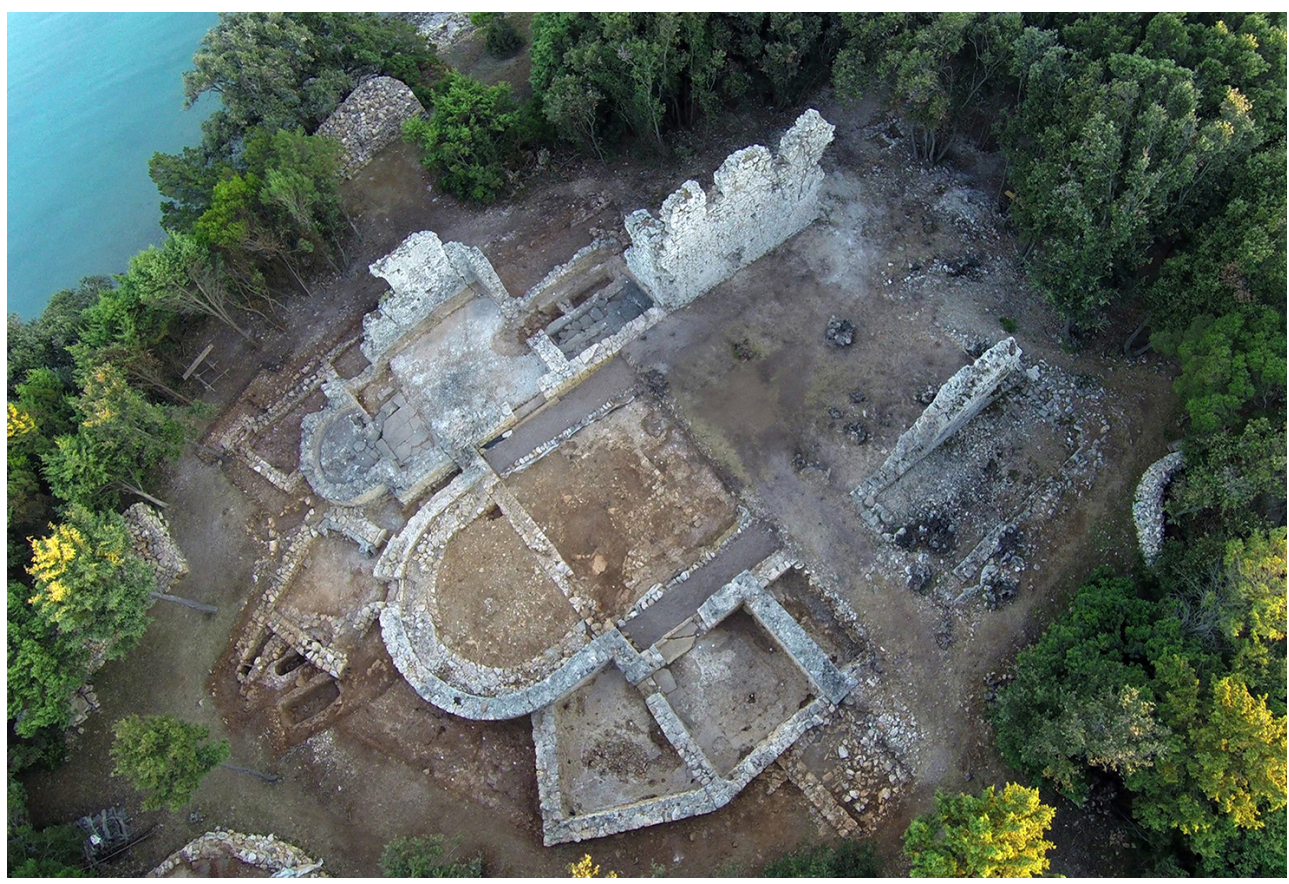

Cl. M. Vuković

Fig. 2 - Démarrage de la restauration des vestiges de la nef, septembre 2017.

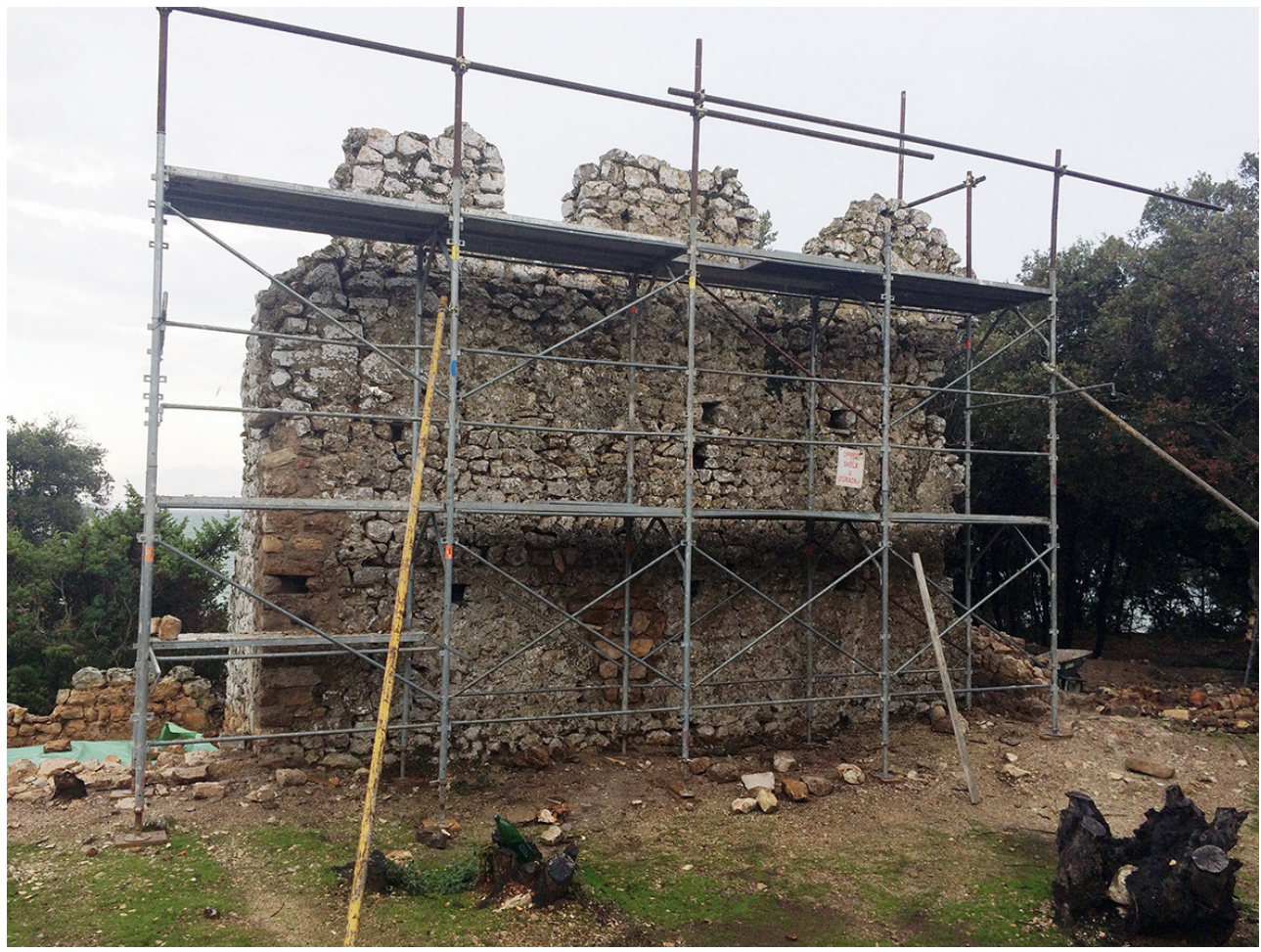

Cl. S. Bully 
Cette année, nous avons testé une datation des mortiers par luminescence optiquement stimulée (OSL), méthode dite "single grain » $^{3}$. Les manipulations (prélèvements et installations de dosimètres) ont été réalisées par Petra Urbanová, archéomètre en postdoctorat à l'UMR IRAMAT-CRPAA de Bordeaux ( $\mathrm{cf}$. infra). La méthode a été appliquée sur l'annexe orientale tardive (Esp. VIIIa) ${ }^{4}$.

\section{Présentation du site et enjeux}

Les premières campagnes de prospection et de relevés topographiques menées entre 2010 et 2012 ont révélé que Martinšćica était un site ecclésial majeur de l'archipel du Kvarner, eu égard à la singularité du parti architectural de sa grande église et des interrogations que suscite le complexe auquel elle appartient - une villa maritime antique et tardo-antique découverte lors des prospections en 2010. Ces premiers résultats ont engagé une poursuite des recherches par des fouilles extensives qui ont débuté sur une partie de la salle de réception de la villa de l'Antiquité tardive en 2014, puis sur la grande église paléochrétienne à partir de 2015. Les campagnes 2015 et 2016 ont ainsi porté sur la fouille de son abside (Esp. Ia), à son chevet (Esp. VIII), dans l'avant-chœur (Esp. Ib), dans une partie du bras nord du transept (Esp. Ic) et dans deux salles annexes au nord (sacristies IIIa et b) (fig. 3).

Fig. 3 - Relevé pierre à pierre général en cours d'élaboration.

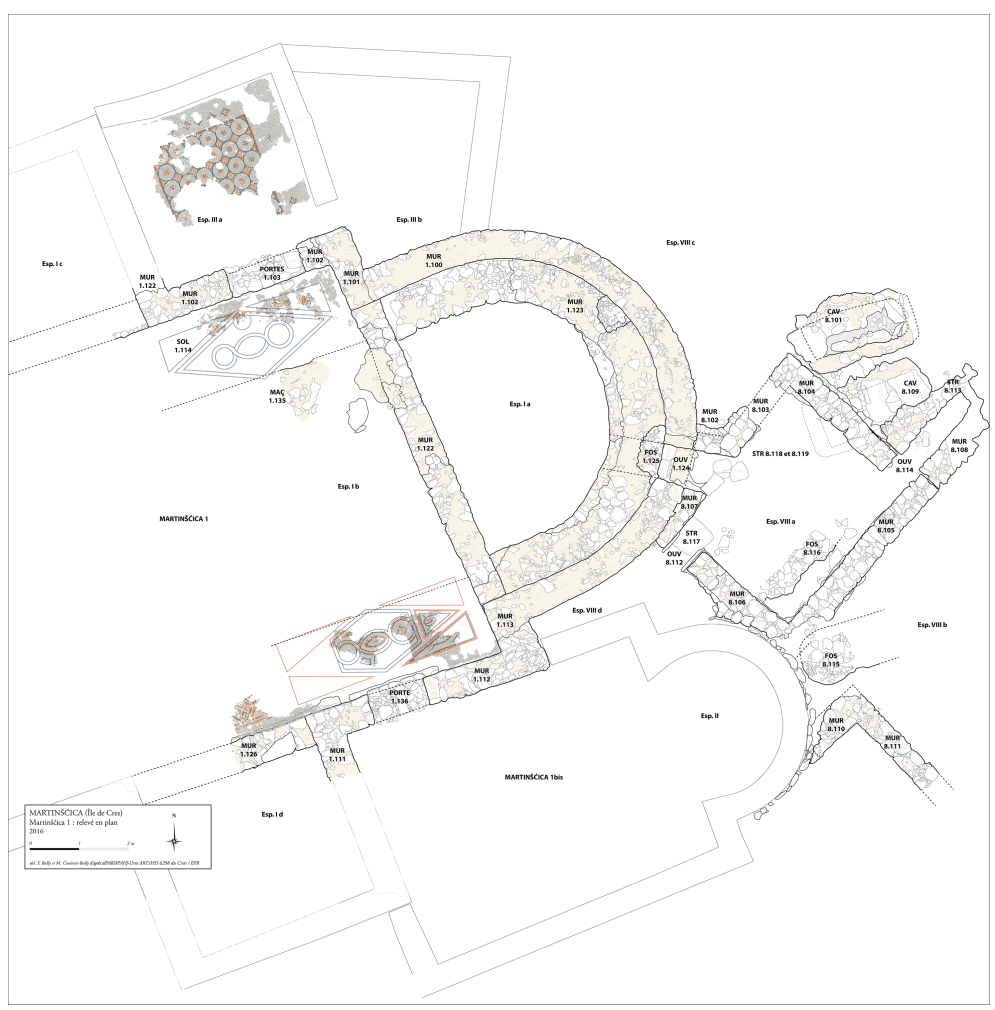

D’après équipe de fouille ; état 2016 avec compléments 2017.

La fouille de l'église doit permettre d'en définir le parti architectural et le phasage et de comprendre la raison de la multiplication des annexes et leur fonction. Il s'agira, à terme, de comprendre également les relations entre le complexe résidentiel (villa) et le 
sanctuaire chrétien dans l'hypothèse de l'installation d'une communauté monastique entre l'Antiquité tardive et le haut Moyen Âge.

\section{Bilan préliminaire de l'étude archéologique de la chapelle latérale sud (Martinšćica 1bis-Esp. II)}

Après la fouille des annexes nord (esp. IIIa et $b$ ) et des parties orientales de la grande église en 2016, l'objectif de la campagne 2017 était de traiter la chapelle à abside outrepassée flanquant la grande église au sud (Martinšćica 1bis). On rappellera que ce type d'édifice à abside outrepassée n'est généralement pas daté au-delà du $\mathrm{VI}^{\mathrm{e}}$ siècle dans la région.

L'opération a débuté par un important travail de défrichement des abords immédiat de la chapelle (fig. 4), suivit d'un terrassement mécanisé sous surveillance archéologique (fig. 5) de la puissante couche de démolition qui avait été reconnue lors du sondage dans l'angle nord-est de la chapelle en 2011. La surveillance du terrassement a permis la découverte de plusieurs nouveaux fragments d'une barrière de chancel du «type de Zadar ", généralement daté de la fin du $\mathrm{V}^{\mathrm{e}}$ siècle-début du $\mathrm{VI}^{\mathrm{e}}$ siècle (cf. rapport 2016). Mais ces fragments appartenaient à la couche de démolition des maçonneries et étaient en situation de matériaux de constructions de la chapelle. Dès lors, la construction du petit édifice à abside outrepassée serait postérieure à la fin du $\mathrm{V}^{\mathrm{e}}$ siècle et pourrait être contemporaine d'une phase de changement du mobilier liturgique de la grande église ou succéder à un édifice antérieur doté de la barrière de chancel du "type de Zadar ". La nature des couches de démolitions laisse supposer que la chapelle était couverte de laves calcaires a contrario de la grande église, qui était couverte de tegulae. On pénétrait dans la chapelle sud par un large passage (1.136) (fig. 6) couvert d'un seuil monolithe qui fut obturé dans une phase déjà avancée de l'abandon et du comblement de la chapelle. 
Fig. 4 - Secteur de la chapelle sud au début des travaux.

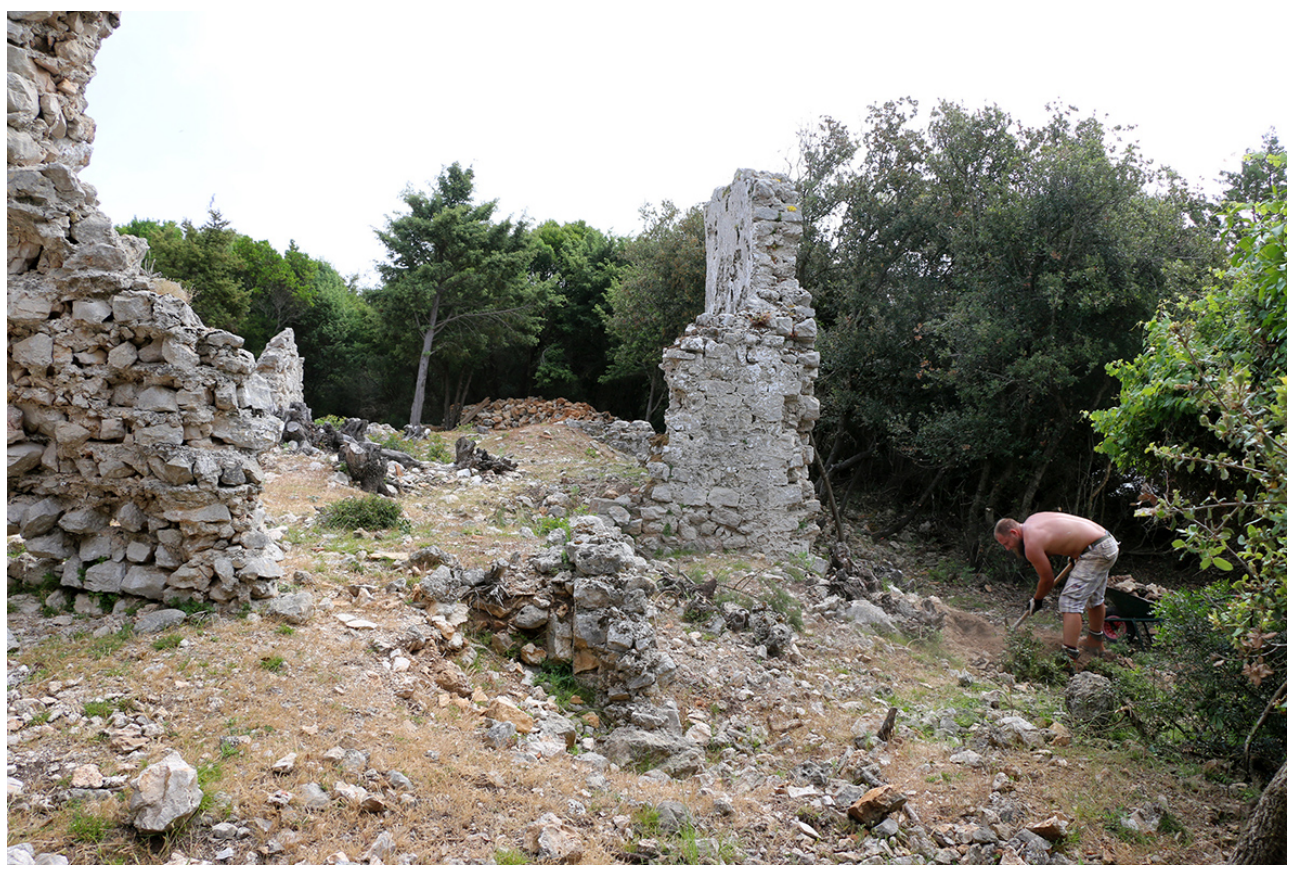

Cl. S. Bully.

Fig. 5 - Travaux de décapage mécanisé à l'intérieur de la chapelle.

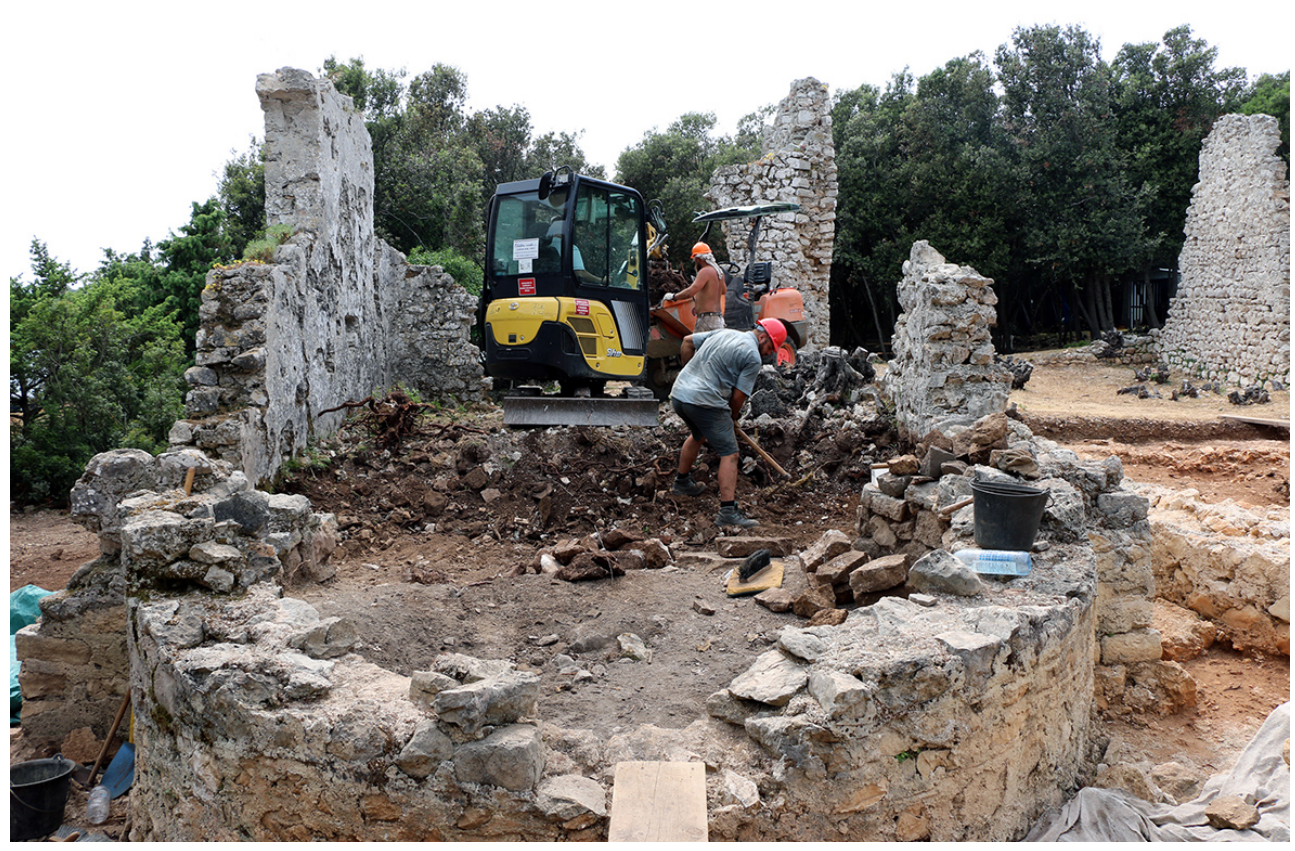

Cl. S. Bully. 
Fig. 6 - Vue de l'ouverture entre l'église et la chapelle sud.

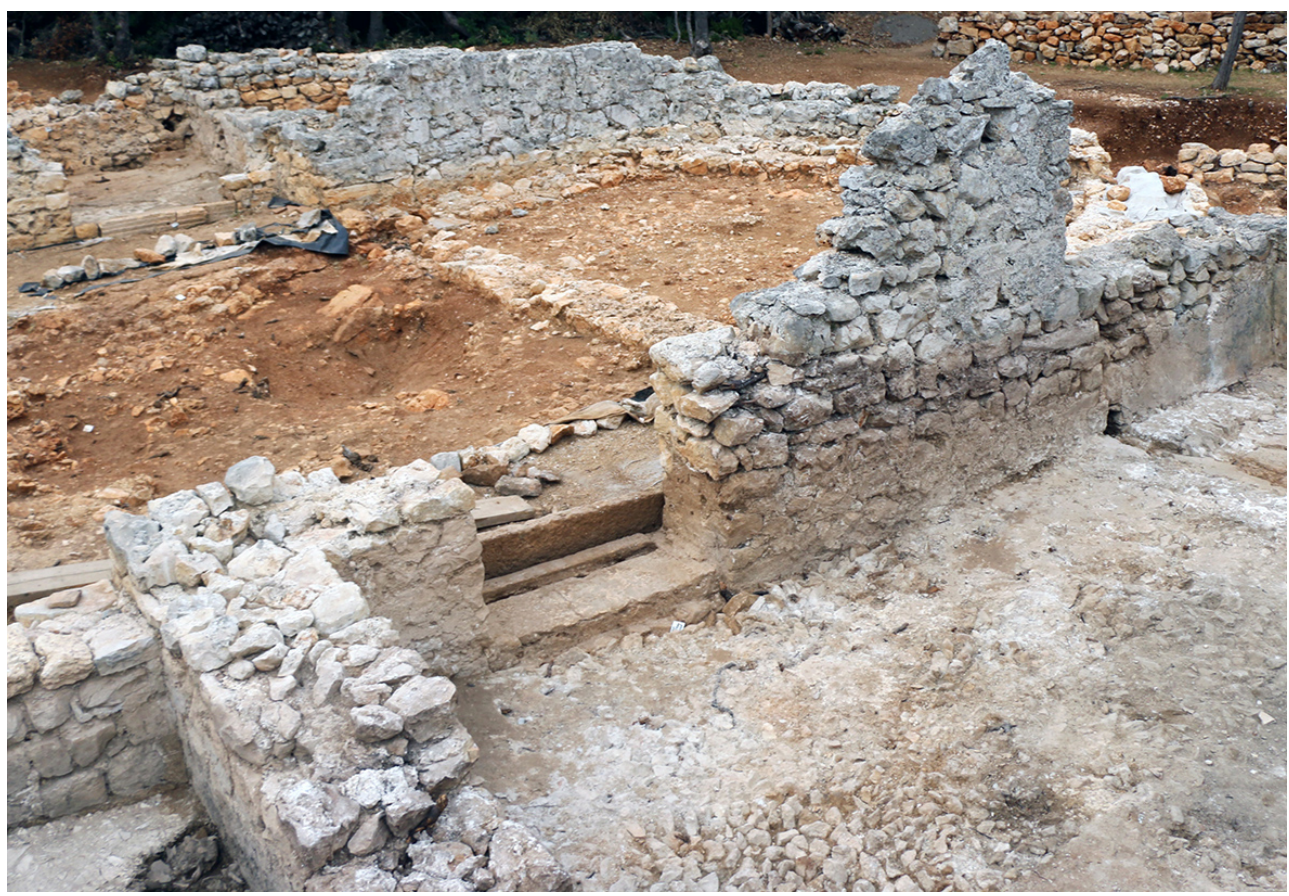

Cl. S. Bully.

Les couches de démolition scellent un niveau de préparation de sol dans la nef particulièrement bien préservé (2.116), formé d'une fine couche de mortier sur un radier de cailloutis (fig. 7). La préparation devait supporter un dallage qui a fait l'objet d'une récupération complète, mais dont l'angle sud-ouest de la nef conserve une trace (2.121) dans le mortier. En revanche, celui-ci est encore en partie présent (2.107) dans l'avant-chœur précédant l'abside 2.100 (fig. 8), séparée de la nef par une barrière de chancel dont subsiste le négatif (2.106). On notera que le niveau de sol de l'avant-chœur était légèrement plus bas - de l'ordre d'une épaisseur de dalle - que le sol de la nef et de l'abside. L'abside est soulignée à sa corde par une marche monolithe précédant un sol (2.111) de mortier et de dalles calcaires irrégulières autour de la base de l'autel (2.112). L'autel est au niveau de la corde de l'abside, légèrement en retrait vers l'est ; sa base est formée par un segment de colonne en remploi d'une trentaine de $\mathrm{cm}$ de diamètre, conservée sur vingtaine de $\mathrm{cm}$ de hauteur (cf. fig. 8). Des négatifs de mortier le long du mur nord de la travée de chœur pourraient être la trace d'un autel secondaire. 
Fig. 7 - Mise au jour des sols à l'intérieur de la chapelle.

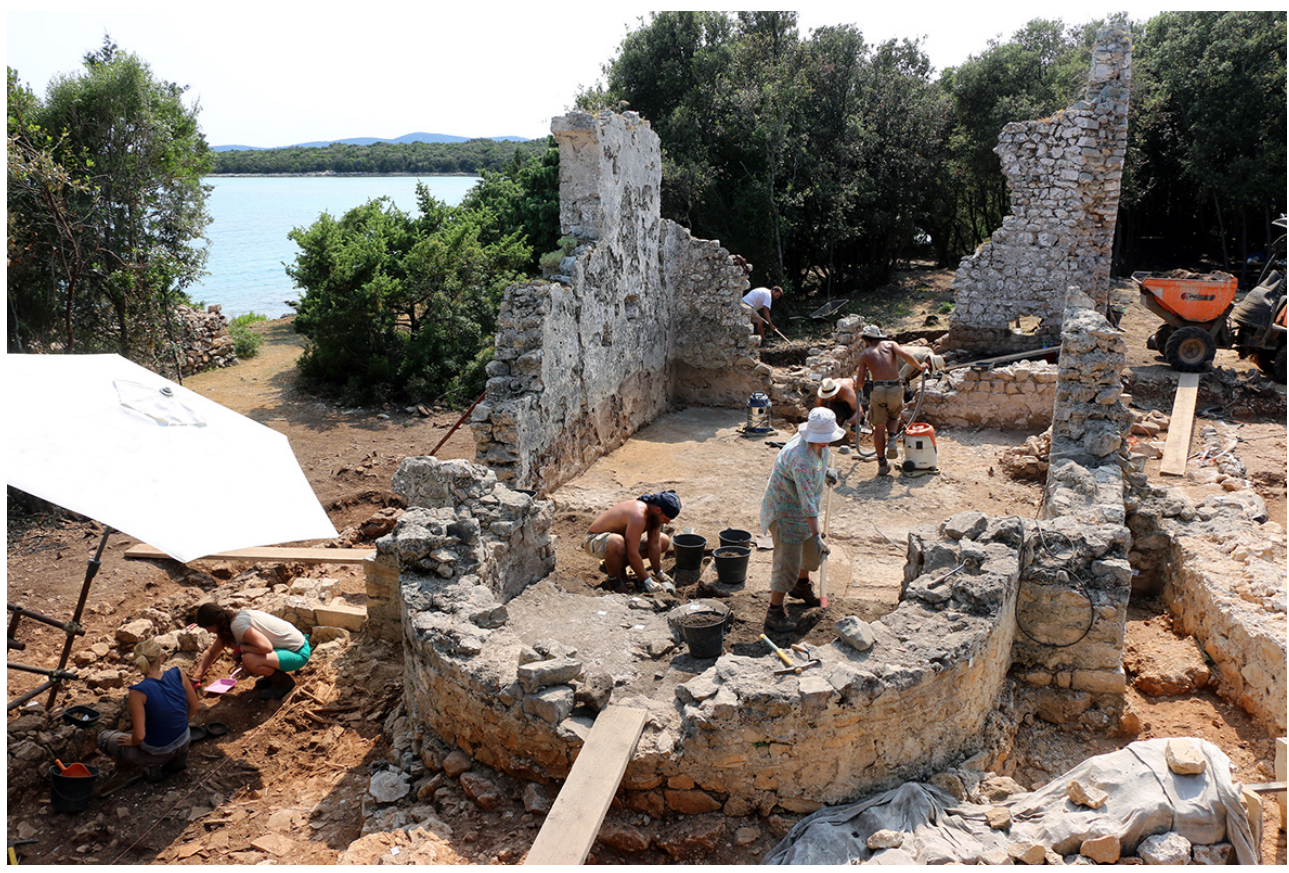

Cl. S. Bully.

Fig. 8 - Vue des sols de l'avant-chœur, de l'abside outrepassée avec la base de l'autel et de l'abside antérieure.

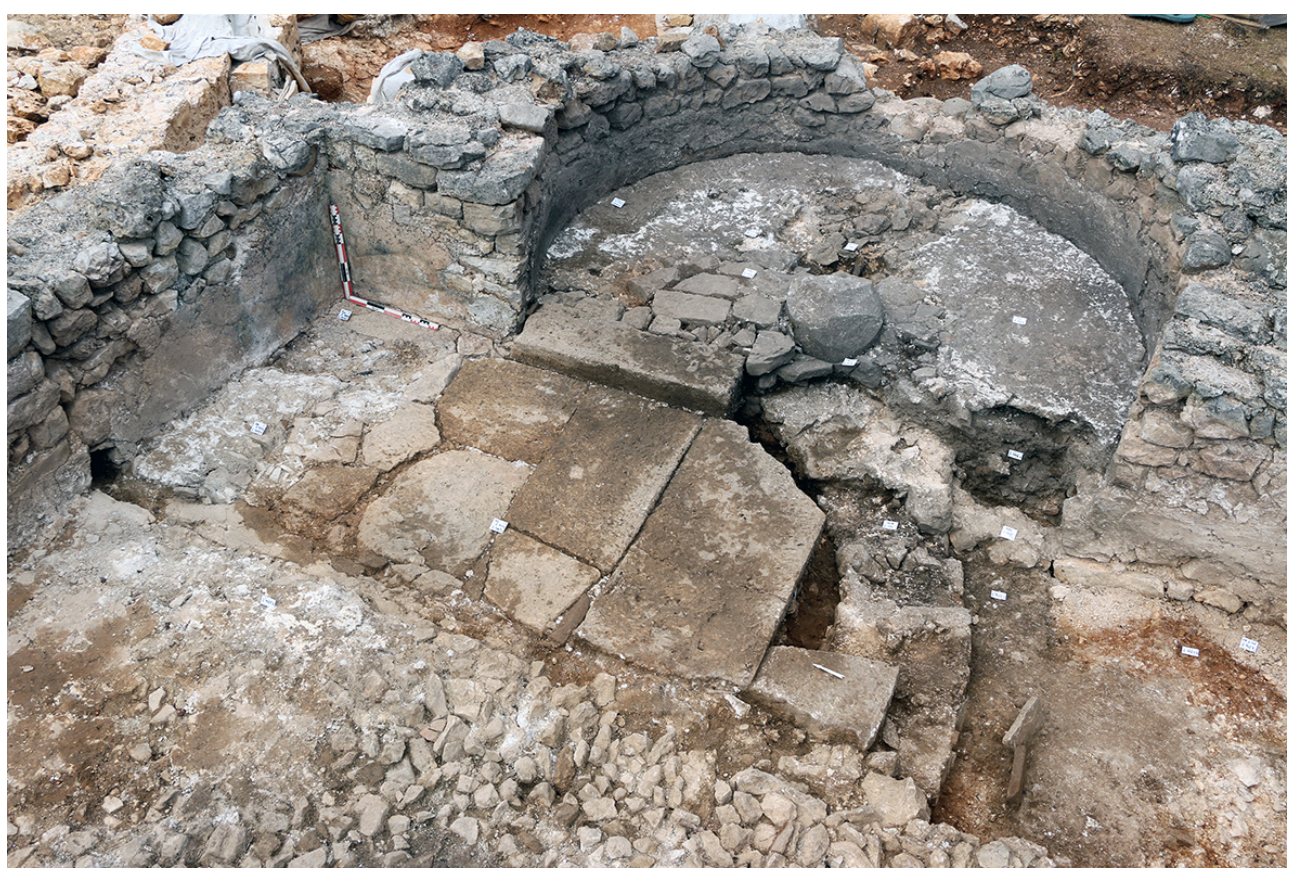

Cl. S. Bully.

Le prélèvement - à une époque indéterminée - du dallage dans la moitié sud de la travée de chœur nous a offert l'opportunité d'un sondage de reconnaissance sous le sol. Ce sondage a rapidement révélé la présence d'une abside antérieure (2.114) (cf. fig. 8-9 ). Il reste hasardeux d'en restituer un tracé - semi-circulaire ou outrepassée, avec ou sans épaulements - et d'en définir sa chronologie relative - comme absolue - avec la 
grande église. On notera cependant qu'une maçonnerie (2.123) orientée est-ouest est apparue sous le sol de la nef, au niveau de l'arrachement du seuil de l'ouverture entre le bras sud du transept et la chapelle. Ce segment de mur est postérieur au parement extérieur du bras sud du transept contre lequel il s'appuie. Seule la poursuite de la fouille permettra de vérifier si ce mur formait ou non le gouttereau sud de l'édifice à abside antérieur.

Fig. 9 - Vue générale de la chapelle sud et des annexes VIIIb et V.

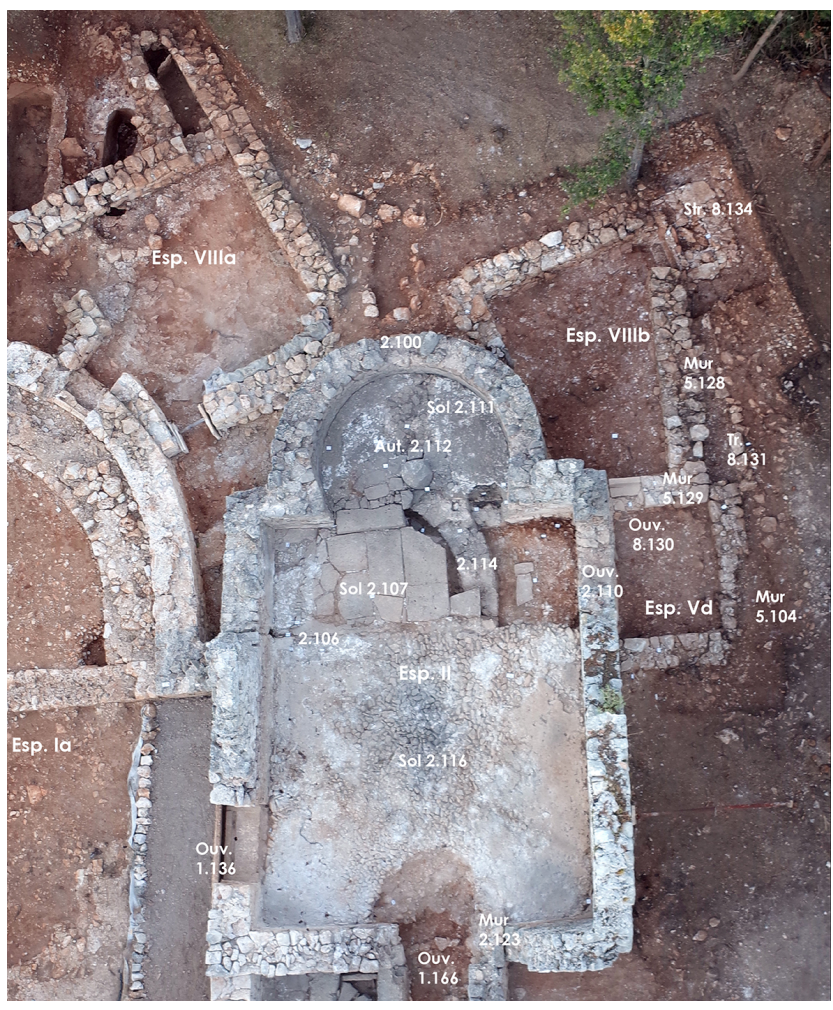

Cl. M. Vuković.

Dans l'attente de sa fouille, on ne se prononcera pas non plus sur la fonction de cet édifice antérieur à la chapelle sud, mais on pressent dans ce secteur la possibilité de dispositifs funéraires et mémoriels. C'est un indice que donneraient l'adoption d'une abside de plan outrepassée pour Martinšćica 1bis mais, surtout, la découverte des tombes privilégiées dans le bras sud du transept (cf. infra).

\section{Bilan préliminaire de l'étude archéologique du bras sud du transept (Esp. Id)}

Au nombre de cinq (1.160 à 1.164), il s'agit de tombes en formae paléochrétiennes d'une très belle facture (fig. 10). Les puissantes maçonneries $( \pm 30 \mathrm{~cm}$ de large) forment des caissons quadrangulaires de dimensions inégales afin d'occuper la totalité de la surface du bras du transept. Les cuves devaient être chacune scellées par une large et épaisse dalle monolithe disposée dans le sens de la longueur et reposant sur les maçonneries des petits côtés. Ceux-ci, moins hauts que les maçonneries latérales, permettaient un parfait ajustement des couvertures avec le sol - disparu - du bras du transept. Seules 
les deux cuves sud (1.160 et 1.164) ont été fouillées, révélant leur curage, peut-être durant le bas Moyen Âge d'après quelques tessons de céramique majolique. Et malheureusement, le comblement apparemment hétérogène, comme les dalles de couvertures brisées ou remplacées par des fragments de dalles irréguliers en guise de sol, laisse craindre qu'il en soit de même pour les trois restantes.

Fig. 10 - Vue générale du bras du transept sud avec ses tombes en forma.

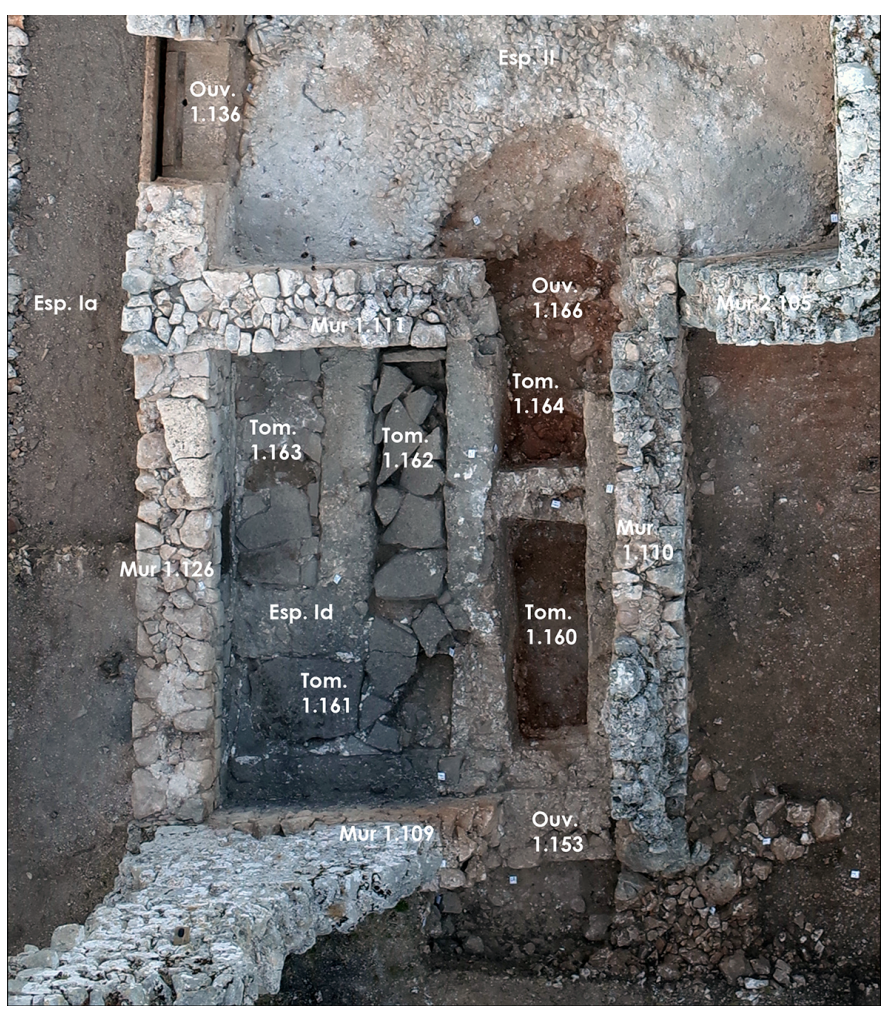

Cl. M. Vuković

Les premières observations - qui demanderont à être confirmées par une analyse plus poussée au moment de la fouille - semblent indiquer que les tombes sont antérieures au mur (1.126) qui ferme le bras du transept sur la nef dans une phase tardive. Dès lors, le bras du transept formerait comme une sorte de vestibule funéraire au-devant de la chapelle à abside outrepassée avec laquelle il communique par une ouverture (1.166) percée dans son mur est (1.111). Dans le cas contraire où les tombes seraient postérieures au cloisonnement du bras du transept, cela signifierait que celui-ci devient une annexe funéraire de la chapelle. On pénétrait également dans le transept sud depuis un passage (1.153) dans son mur ouest (1.109). Il s'agit là des points essentiels sur lesquels nous reviendrons lors de la prochaine campagne. Mais à titre d'hypothèse de travail, nous suggérons que la présence de tombes en formae dans ce secteur pourrait être liée à celle d'une tombe importante sous la chapelle à abside outrepassée ou de reliques qui étaient présentées dans celle-ci (hypothèse plus difficilement vérifiable...). Notons encore qu'une sépulture d'enfant a été tardivement déposée contre le mur est (1.111) du transept, dans une fosse creusée dans les niveaux de démolition et d'abandon de l'église. 


\section{Bilan préliminaire de l'étude archéologique des annexes VIIIb et Vd (fig. 9)}

Dès les travaux de terrassement au chevet de l'église, en 2015, nous avions découvert une nouvelle annexe (VIIIb) greffée sur le flanc sud de l'abside outrepassée de la chapelle I bis. Cette dernière campagne en a permis la fouille quasi totale, révélant que, dans son dernier état, la salle VIIIb était précédée d'un petit vestibule à l'ouest (Vd) et dotée d'un petit aménagement (8.134) dans son angle sud-est.

Les vestiges très arasés des deux salles sont apparus immédiatement sous les couches d'humus et de démolition de l'église (fig. 11). On pénétrait dans la salle occidentale $\mathrm{Vd}$ depuis la chapelle sud à travers une ouverture (2.110) percée au niveau de l'avantchœur (matérialisé par le sol dallé). Le petit vestibule $\mathrm{Vd}$ desservait la salle principale VIII b par une étroite ouverture (8.130) dotée d'un fragment de seuil en remploi. L'étroitesse du passage $( \pm 0,52 \mathrm{~m})$ renvoie à celle des ouvertures de l'annexe VIIIa et des sacristies IIIa et $b$ dans leurs phases tardives. Mais l'aménagement du vestibule $\mathrm{Vd}$ résulte d'une subdivision d'un espace formé à l'origine d'une seule salle (VIIIb). Cette transformation est clairement visible dans la modification du tracé du mur sud 5.104 qui s'interrompt, dans une articulation maladroite, au niveau du mur de refend 8.129 ; celui-ci se poursuit ensuite à angle droit avec le mur 8.128 pour s'ajuster sur la structure d'angle 8.134. Le tracé primitif du mur sud-est cependant clairement restituable par la reconnaissance de sa tranchée de fondation-épierrement 8.131.

Fig. 11 - Mise au jour des annexes VIIIb et Vd.

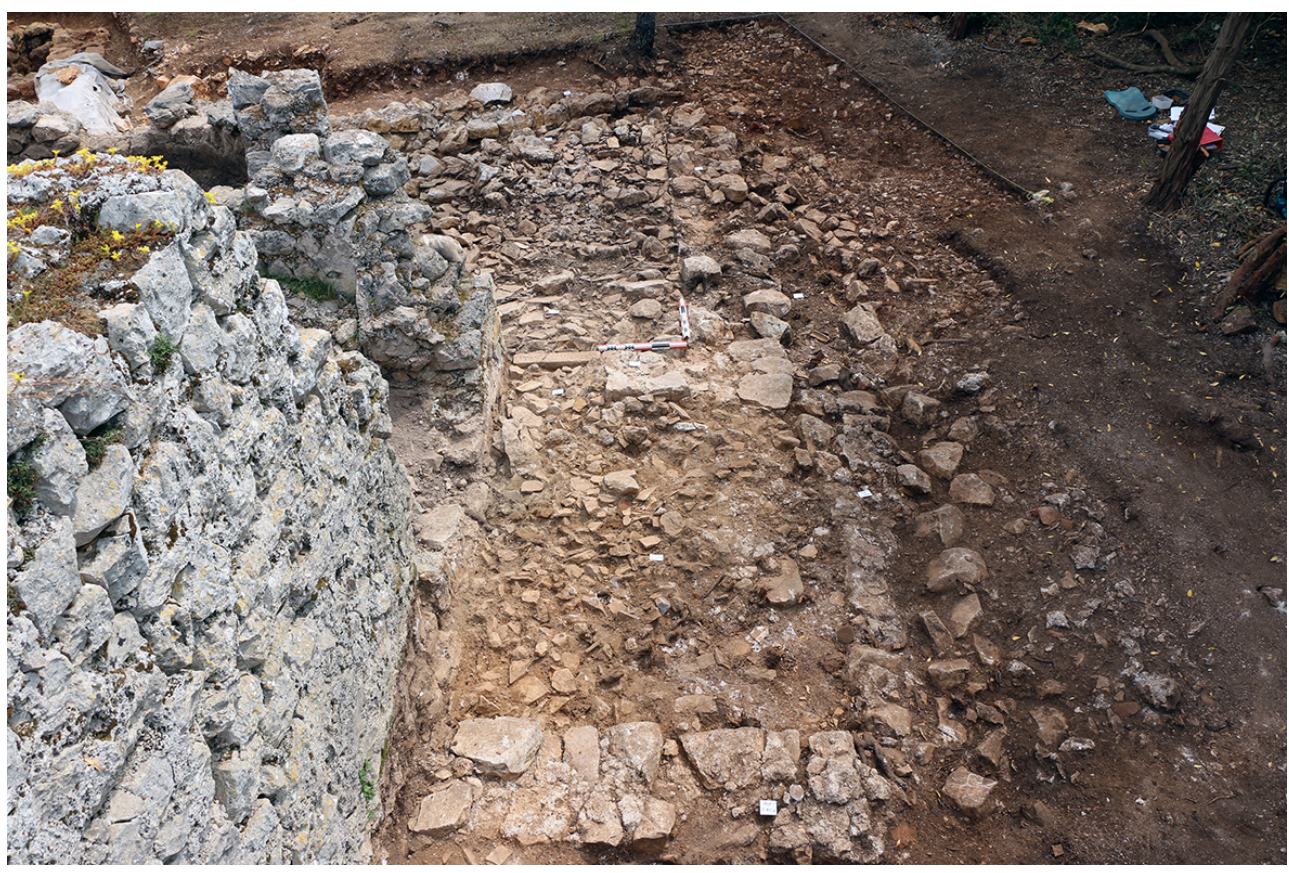

Cl. I. Valent

Le comblement supérieur des deux salles était constitué de couches d'abandon et de démolition de leurs toitures de laves et de l'effondrement de leurs murs. En revanche, la salle VIIIb était également en partie comblée sur toute sa surface par une couche très hétérogène d'ossements humains mélangés à des laves calcaires (fig. 12). L'étude 
anthropologique de ces ossements est en cours. Sans que nous puissions le certifier, nous nous demandons si cet ossuaire ne serait pas issu du curage tardif (au bas Moyen Âge ?) des tombes en formae du bras du transept. Le dernier niveau de sol du vestibule $\mathrm{Vd}$ a en effet livré de la céramique majolique, comme c'est le cas dans les niveaux d'abandon du transept sud.

Fig. 12 - Vue de la couche de curage d'ossements humains dans l'annexe VIIIb.

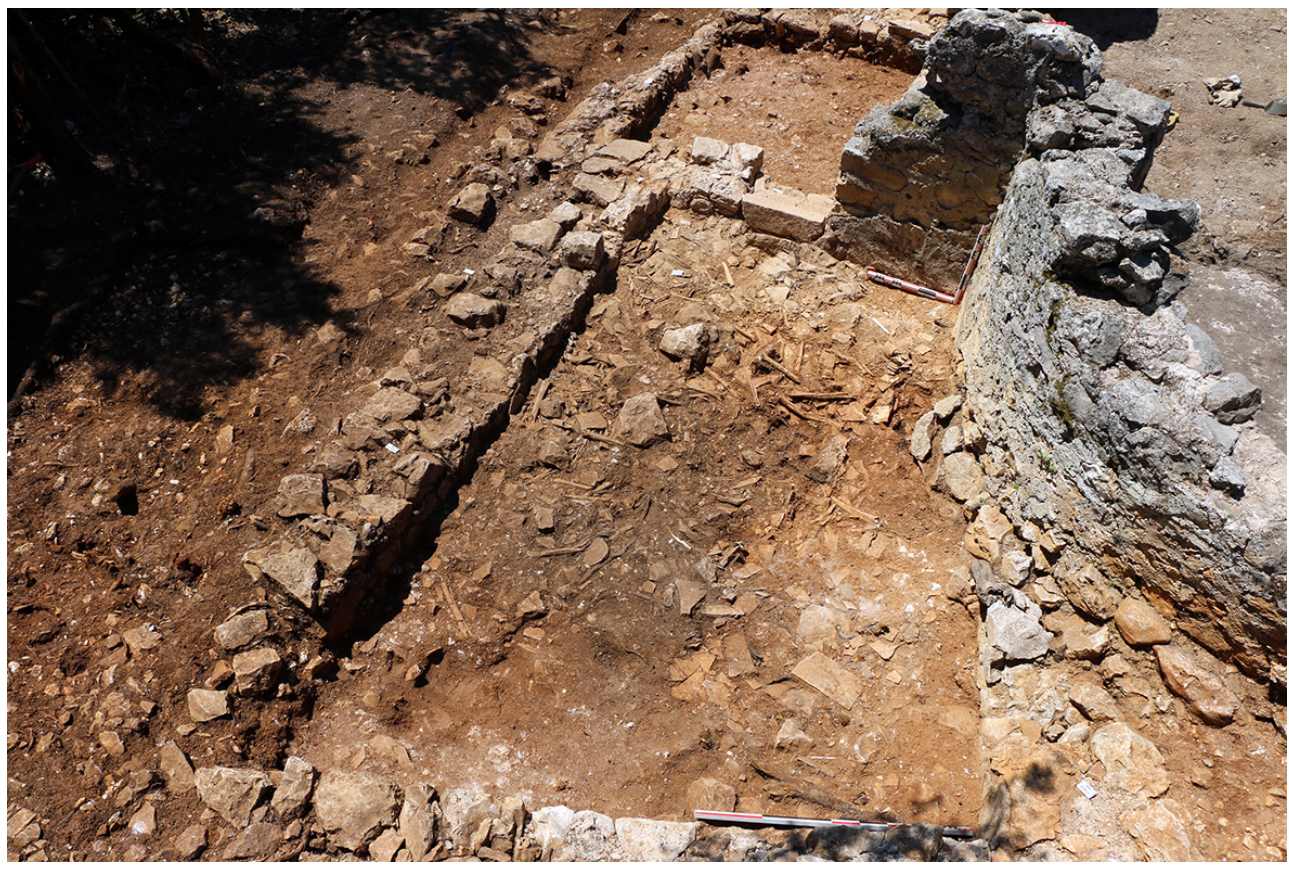

Cl. S. Bully.

Les frustes sols d'origines des deux salles, formés d'un mélange d'argile et d'un mortier pauvre en chaux, n'ont pas conservé de vestiges significatifs de l'usage de ces pièces, à l'exception, peut-être, de restes de consommation de faune. En revanche, nous espérons beaucoup de la structure 8.134 dans l'angle sud-est de la salle VIIIb (fig. 13). Celle-ci ayant été découverte très tardivement, nous avons fait le choix de ne pas la fouiller dans la précipitation, afin de ne pas passer à côté d'indices déterminants pour la compréhension de la fonction de ces espaces. La structure se présente comme un petit augmentum situé dans le prolongement du mur oriental 8.111. On y accède par une étroite ouverture (8.133) accueillant les négatifs de mortier lissé de deux petites marches monolithes - disparues (fig. 14). Les murs périphériques de la structure forment les limites d'un aménagement interne de plan quadrangulaire, réduit sur son petit côté ouest dans un premier temps avant d'être comblé. Tout en se présentant différemment, ce dispositif n'est pas sans évoquer celui déjà rencontré pour l'annexe VIIIa et que nous identifions actuellement comme une latrine ou un lieu de stockage. La poursuite de la fouille devra nous assurer cependant que la structure 8.134 n'est pas uniquement le seuil maçonné d'une entrée extérieure dans la salle VIIIb. 
Fig. 13 - Vue générale des annexes VIIIb et Vd.

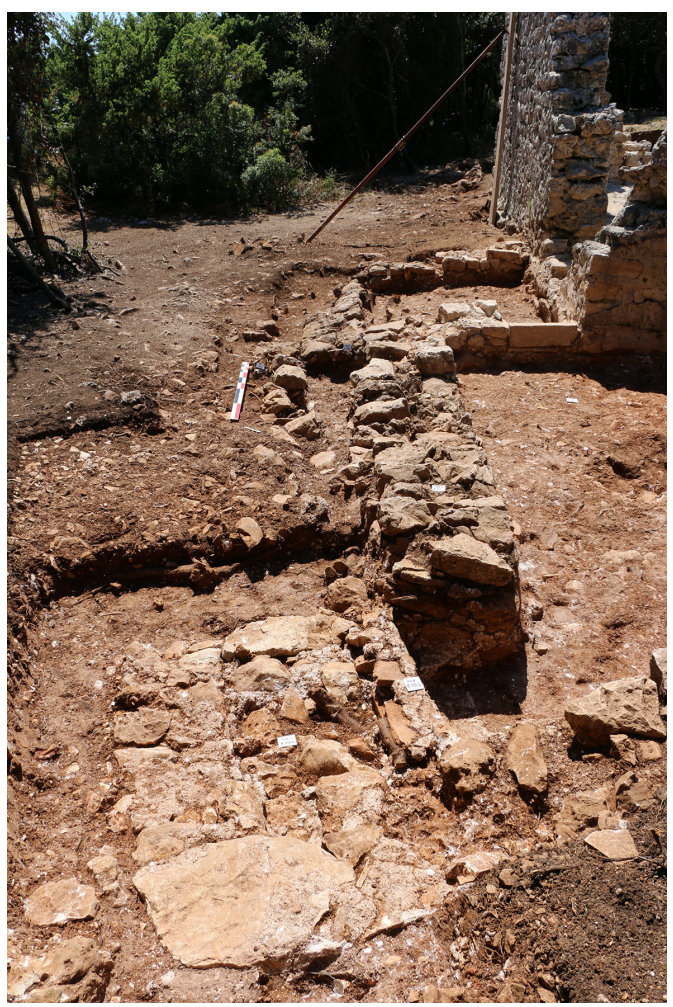

Cl. I. Valent.

Fig. 14 - Détail de la structure 8.134 de l'annexe VIIIb.

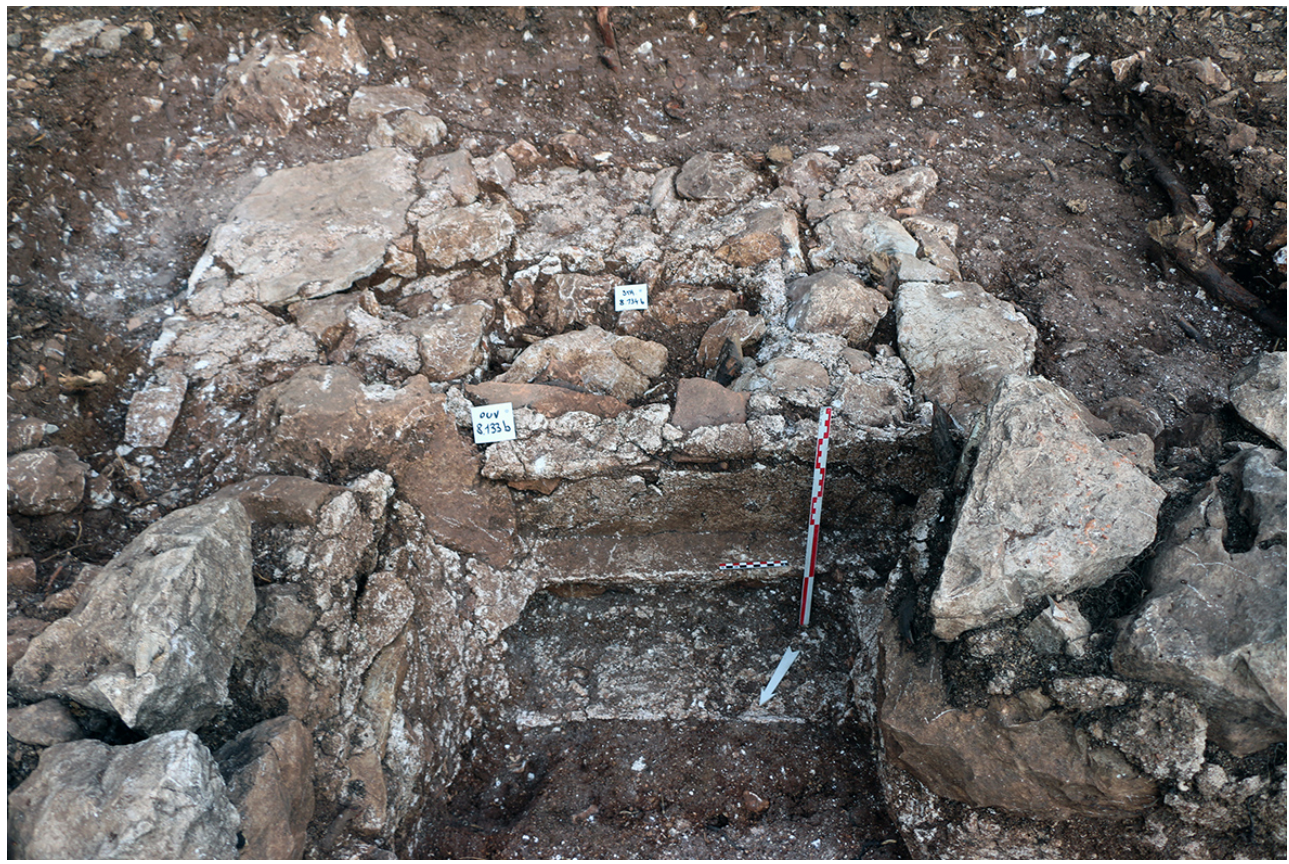

Cl. S. Bully.

Les grandes orientations des annexes VIIIa (fouillée en 2015-2016) et VIIIb, comme le mode de construction et la stratigraphie plaident fortement en faveur de leur contemporanéité. Et l'analyse du mortier du mur 8.104 de l'annexe VIIIa par la méthode 
SG-OSL (cf. infra) confirme notre hypothèse d'une construction haut-médiévale avec une datation vraisemblable dans le VIII ${ }^{e}$ siècle. Cette datation demande cependant à être corrélée avec les analyses $\mathrm{C}^{14}$ en cours et l'étude du mobilier céramique.

\section{Complément à l'étude archéologique de l'annexe IIla et de l'espace VIIIc}

La sacristie IIIa logée dans l'angle formé par le transept nord et l'avant-chœur a fait l'objet de compléments de sa documentation - avec le relevé de sa mosaïque (cf. fig. 3) et de fouilles. Il s'agit principalement de la fouille du niveau cendreux (3.1014) qui recouvrait une structure foyère (3.117) marquée par un impact thermique sur le sol et les murs (fig. 15). La fouille fine du sol a en outre révélé que la structure foyère était délimitée par un alignement légèrement circulaire de 4 trous de piquets inscrits dans le sol de mortier 3.110 (fig. 16). Ce dispositif n'est pas sans rappeler l'aménagement tardif du lavabo 3.111 de la sacristie IIIb (cf. chroniques 2016). Des analyses radiocarbones sur des charbons de bois prélevés dans la couche cendreuse permettront de dater cette phase d'occupation - domestique? - de la sacristie.

Fig. 15 - Vue générale de la sacristie Illa avec son sol de mosaïque et sa structure foyère.

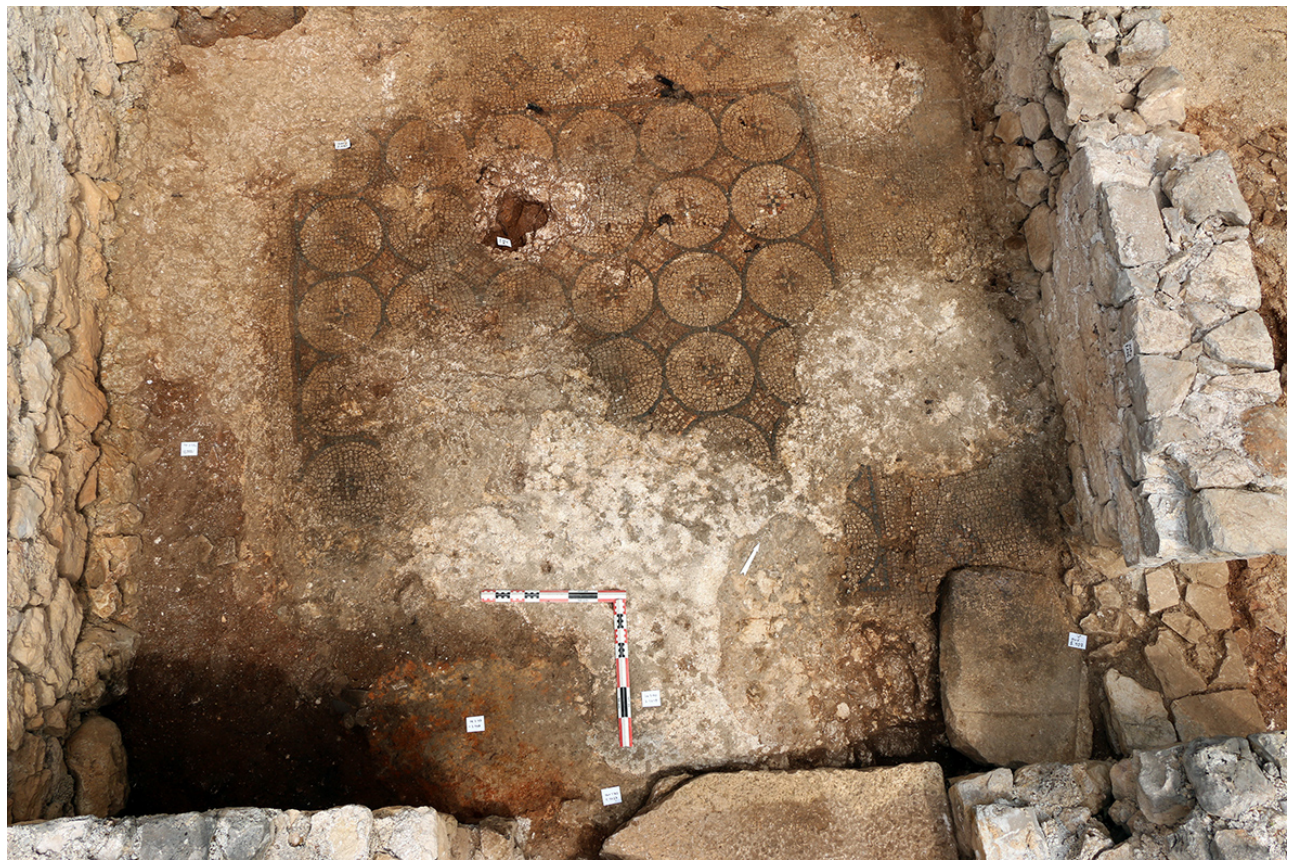

Cl. S. Bully. 
Fig. 16 - Détail de la structure foyère 3.117 de la sacristie IIla.

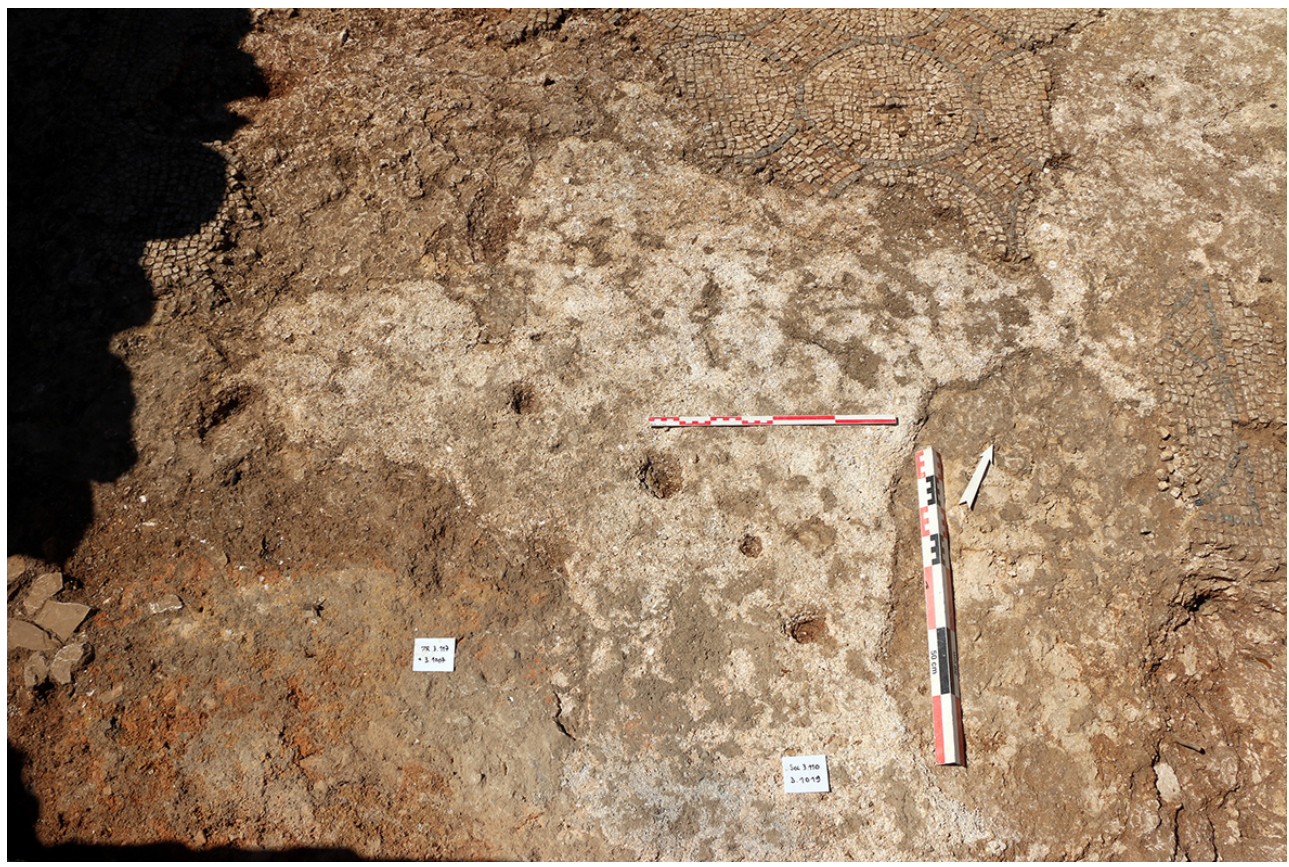

Cl. S. Bully.

L'annexe orientale VIIIa et son dispositif 8.113 recouvraient une tombe de l'Antiquité tardive (8.109) encore en grande partie scellée par une couverture de dalles calcaires noyées dans un abondant mortier. Son ouverture a révélé une cuve enduite d'un mortier de couleur rouge clair. La cuve adopte un plan particulier avec ses deux extrémités semi-circulaires. Elle était en partie comblée par des blocs volontairement déposés en remplissage de la tombe au moment de la construction du mur oriental 8.104 de l'annexe VIIIa (fig. 17). Les blocs recouvraient une couche d'ossements en position secondaire, probablement engendrée par la perturbation. Mais à l'issue de la fouille, il apparaît que la tombe contenait au moins trois individus en situation primaire. L'étude anthropologique permettra de statuer sur le nombre exact d'individus inhumé dans cette tombe et sur leur sexe. Rappelons que dans la proche tombe 8.101 il s'agissait de trois individus masculins. 
Fig. 17 - Vue générale de la tombe paléochrétienne 8.109 en cours de fouille.

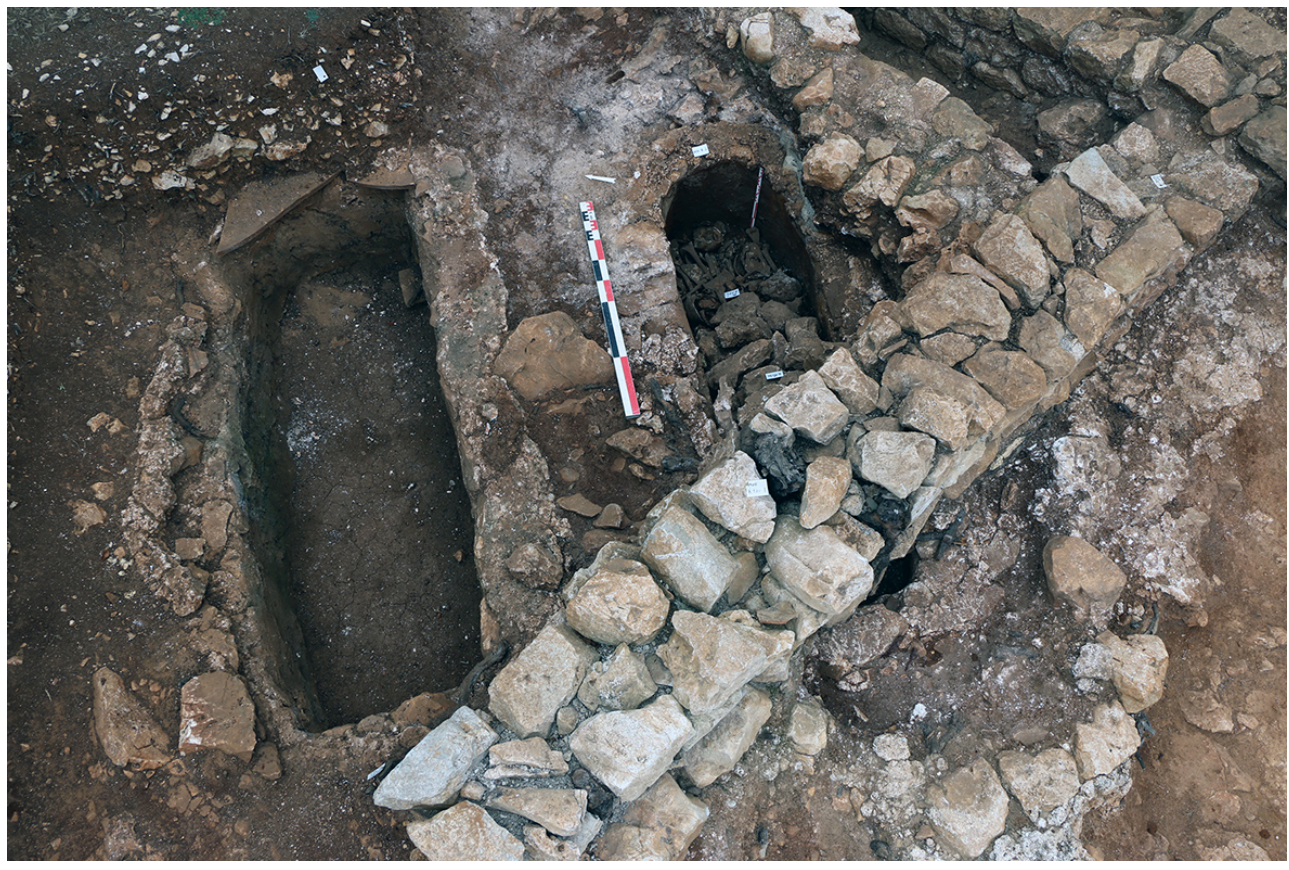

Cl. L. Dugorepec.

\section{Découverte fortuite d'un sceau byzantin (V. Prigent)}

L'équipe archéologique a été contactée au début du mois de septembre par M. Tomaž Hartman qui avait découvert fortuitement un sceau en plomb (fig. 18) à l'occasion d'une visite du site au printemps $2017^{5}$. Le sceau a été trouvé sur la grève à quelques mètres de la grande église. Une première expertise révèle qu'il s'agit d'un très beau sceau byzantin du début du VIII ${ }^{\mathrm{e}}$ siècle d'un notaire impérial nommé Pardos ${ }^{6}$.

Fig. 18 - Sceau byzantin (PO.MAR.2017-6).
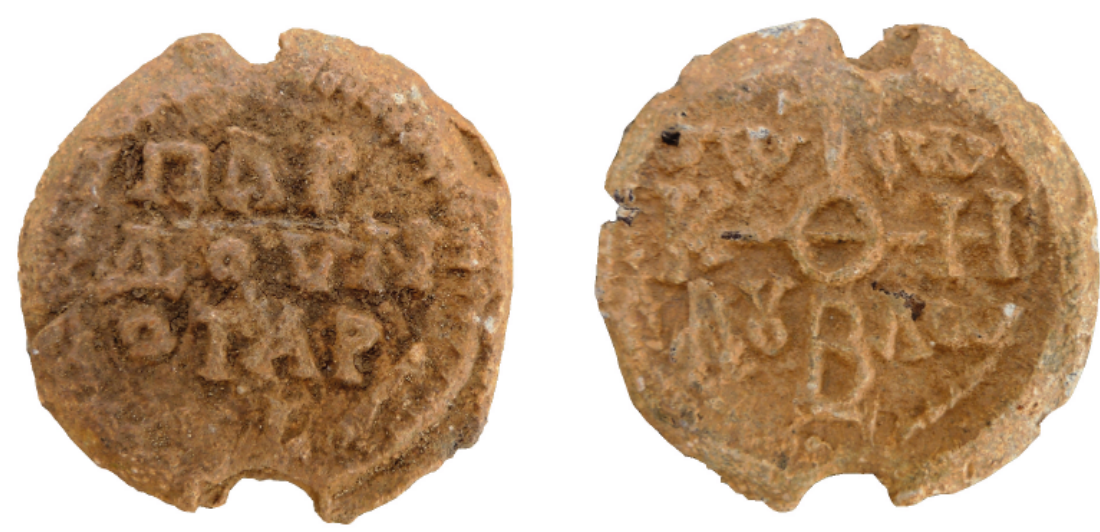

Cl. Tomaž Hartman 
Édition diplomatique

PARDOS, NOTARIOS

Date : début du VIII ${ }^{\mathrm{e}}$ siècle

Parallèle : aucun

Diamètre : 25,5 mm

Poids : $13,76 \mathrm{~g}$

AVERS: bordure de feuillage stylisée. Monogramme invocatoire de type Laurent V

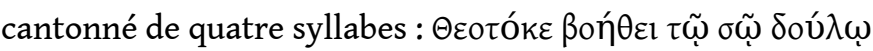

REVERS : bordure de feuillage stylisée. Légende sur trois lignes, suivie d'une croisette sur une ligne autonome

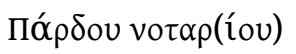

\section{La mensa d'autel et son signum monogrammatique ( $P$. Chevalier)}

Un angle de mensa d'autel en marbre blanc (LAP.MAR 16.24) a été découvert en 2016 dans l'espace III (fig. 19). Il s'agit d'un plateau à rebord épais sans doute rectangulaire d'une typologie des plus classiques, lisse et poli sur les faces visibles, très finement dressé au revers. Les tranches concaves sont terminées aux angles par des lunules permettant que l'arête soit verticale et pleine. La bordure moulurée se compose d'une plate-bande périmétrique large de $3,5 \mathrm{~cm}$, suivi d'un anglet évasé que forment un filet oblique descendant lié à un bandeau en retrait et oblique contraire large de $2 \mathrm{~cm}$. Un cavet assez raide cerne ensuite le champ central, ainsi creusé en cuvette sur $3,2 \mathrm{~cm}$ au minimum ; la cassure empêche en effet de distinguer si une tablette basse terminée par un filet limitait le centre du plateau et en diminuait encore la faible épaisseur $(1,6 \mathrm{~cm}$ à la cassure). L'originalité de ce petit fragment $(9 \times 11 \mathrm{~cm}$ max. cons.; ép. max. $4,8 \mathrm{~cm})$ tient à la présence d'un monogramme gravé assez profondément au revers, à un emplacement - proche d'un angle de la mensa - que l'on retrouve sur la plupart des exemples comparables. 
Fig. 19 - Fragment d'autel avec monogramme.

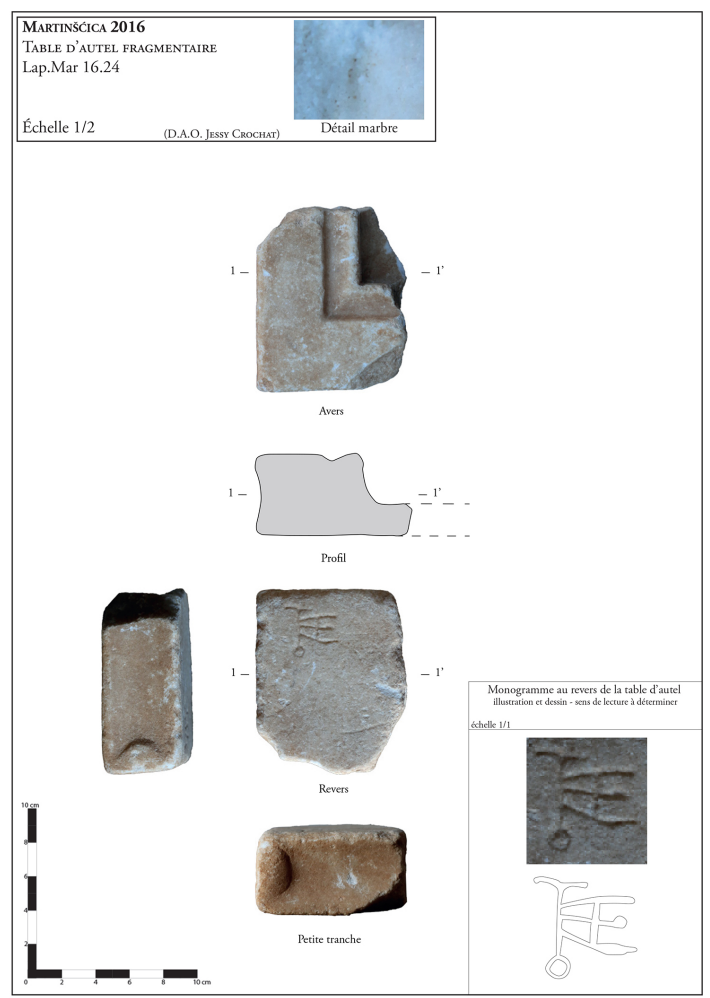

Cl. et dessin J. Crochat.

Si l'on considère que l'on a affaire à un monogramme bloc formant un nom (et un titre ?) en lettres latines, il peut être décomposé comme suit : la longue haste d'un $\mathrm{T}$ à traverse très légèrement bifide semble posée sur un petit $O$, lui sont attachées ou suspendues deux barres un peu obliques et évasées d'un A trapézoïdal à traverse droite ; une troisième ligne médiane vient former un $\mathrm{E}$ sous la traverse $\mathrm{du} \mathrm{A}$. On peut en outre retrouver (et lire) d'autres lettres comme le C (dans le O), le V (dans le A), le F et le $\mathrm{L}$ (dans le $\mathrm{E}$ ), ainsi que le I dans la barre médiane ou ailleurs, et peut-être encore un $\mathrm{H}$ un peu trapézoïdal dans le A. On aurait donc possiblement par ordre alphabétique les lettres A, C, E, F, H, I, L, O, T et V. Le S absent ne facilite pas la restitution d'un nom masculin au nominatif, mais il pourrait être au génitif.

$\mathrm{Si}$, en revanche, on pense à un monogramme grec - et le marbre blanc à gros grain pourrait suggérer une provenance orientale - on considérera la décomposition suivante, nettement plus riche de possibilités : la longue haste d'un tau à traverse très légèrement bifide semble posée sur un petit omicron, lui sont suspendues deux barres faiblement obliques et évasées d'un alpha trapézoïdal à traverse droite; une troisième ligne médiane vient former un omega minuscule $(\omega)$ angulaire et tête bêche au-dessus de la traverse de l'alpha, puis un epsilon sous la traverse de l'alpha. On peut en outre retrouver d'autres lettres comme le sigma (С) (dans l'omicron), le gamma (Г) et le pi (П) (dans l'epsilon), ainsi que le iota dans la barre médiane ou ailleurs, mais aussi un chi (X) dans la croix formée par la barre médiane et la traverse de l'alpha, un delta $(\Delta)$ dans une moitié de l'oméga, et peut-être encore un eta $(\mathrm{H})$ et un lambda $(\Lambda)$ un peu trapézoïdal dans l'alpha. La forme bifide du tau pourrait enfin éventuellement être vue comme un upsilon très plat. Les lettres discernables seraient donc, toujours dans l'ordre 
alphabétique, A Г $\triangle \mathrm{E} H \mathrm{H}$ I $\Lambda$ O П С T $\Upsilon \mathrm{X}$ majuscule et $\omega$ minuscule comme souvent. Un nom masculin en -oৎ ou un génitif en -ov seraient par conséquent également possibles.

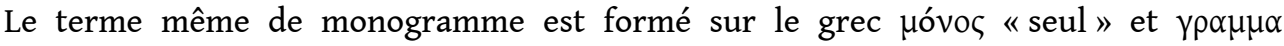
"lettre». Afin de caractériser ce signum complexe, Robert Favreau, reprend la définition énoncée par Raban Maur dans son De inventione linguarum ${ }^{7}$ : le monogramme rassemble unum characterum cum congerie litterarum, autrement dit "sous un même graphisme un groupe de lettres $»^{8}$. Reprenant déjà cette même idée dans son Manuel de Diplomatique ${ }^{9}$, A. Giry le qualifiait $\mathrm{d}^{\prime}$ " assemblage de plusieurs lettres, conjointes et entrelacées de manière à ne former qu'un seul caractère ", même si quelques lettres sont parfois séparées de l'armature du monogramme, tant et si bien que le signe obtenu " contient les éléments d'un ou de plusieurs mots", selon les termes de M. Deloche ${ }^{10}$. Lorsque le mot est unique, c'est presque toujours un nom propre, ainsi que le rappelait H. Leclercq dans le Dictionnaire d'archéologie chrétienne et de liturgie ${ }^{11}$. Selon la définition offerte plus récemment par Jean-Claude Cheynet, le monogramme est une " combinaison de lettres dont le déchiffrement donne un nom, une titulature [...]. Aucun ordre dans la disposition ne permet de faciliter le déchiffrement de ces éléments ${ }^{12}$. [...] On distingue deux types de monogrammes, le plus ancien, compact, construit autour d'une lettre et l'autre cruciforme qui apparaît au $\mathrm{VI}^{\mathrm{e}}$ siècle. Les chapiteaux de Sainte-Sophie (avant 537) au monogramme de Théodora ${ }^{13}$ sont le premier exemple connu. » Robert Favreau souligne que ces "schémas abréviatifs », ces formes ramassées du ou des mots véhiculent une charge de sacralité que l'on peut rapprocher des nomina sacra comme le Chrisme ou la croix monogrammatique. Ce sont donc des nomina porteurs de sens ${ }^{14}$.

Pour employer un vocabulaire actuel, anachronique mais parlant, le monogramme est un «logotype » ou «logo ", une "identité visuelle " comme en créent de nos jours à foison les designers graphiques, où la distribution des $\mathrm{X}$ lettres qui le composent condense et articule textuel et visuel. Et c'est logiquement sur les bagues sigillaires et sceaux ${ }^{15}$ autant que sur les monnaies - mediums par excellence de la publicité de nomina importants, au premier titre desquels figure celui de l'empereur - que l'on trouve le plus grand nombre de monogrammes nominatifs à la fin de l'Antiquité.

L'usage dans l'architecture du monogramme-bloc ${ }^{16}$ - en allemand « Blockmonogramm ${ }^{17}$ ", en anglais « box (type) monogram » - par un évêque commanditaire est attesté dans le troisième quart du $\mathrm{V}^{\mathrm{e}}$ siècle, en l'occurrence par Néon de Ravenne (v. 450-v. 474) ${ }^{18}$. Les occurrences se font plus nombreuses à la fin $d u V^{e}$ et au VI ${ }^{e}$ siècle de Rome à Constantinople, avant qu'elles ne se raréfient et que ne vienne le supplanter en Orient ${ }^{19}$ après les règnes de Maurice (582-602) et d'Héraclius (602-610) le monogramme cruciforme ${ }^{20}$ à la longue descendance médiévale ${ }^{21}$, apparu quant à lui au $\mathrm{VI}^{\mathrm{e}}$ siècle (entre 518 et $533^{22}$ ).

On connaît un certain nombre de ces monogrammes-blocs gravés au revers de mensae d'autel en marbre, comme ici à Martinšćica. Tous les exemples de comparaisons que nous avons pu recenser à ce jour lors d'observations et d'études d'objets ou dans la bibliographie apparaissent être des monogrammes grecs. Ainsi, en Albanie actuelle, sur deux exemples de Byllis et un plateau de Saranda ${ }^{23}$, le monogramme - supposé être celui d'un évêque (Praesios à Byllis et Paulos à Saranda) - a été gravé entre une croix et une lettre (sigma et chi à Byllis, mu à Saranda) qui paraît être un guide de pose correspondant à une lettre gravée sur la colonnette la plus proche de l'angle où monogrammes et signes sont gravés. C'est en tout cas ce qui a pu être démontré pour 
l'autel de la basilique A de Byllis, où les plinthes des bases des colonnettes portent une lettre (bèta, sigma, etc. ${ }^{24}$. L'exemplaire de Saranda est en tous points identique au monogramme trouvé au martyrium d'Akrotiri (Chypre) ${ }^{25}$. Plus rares sont les monogrammes gravés sur la plate-bande périphérique de la moulure, a fortiori quand ils sont multiples comme sur un plateau de Saint-Isidore de $\mathrm{Chios}^{26}$.

Deux interprétations s'opposent généralement pour ces signes lapidaires monogrammatiques : on y devinerait, tant ils sont difficiles à réellement lire, soit le nom du commanditaire, éventuellement l'évêque appelé aussi à consacrer l'autel, soit celui du marbrier. Si l'on admet qu'il s'agit du nom discrètement gravé du donateur, qui pouvait être un évêque, notamment lorsque la table est celle d'une cathédrale, ou celui de l'évêque consécrateur éventuellement aussi donateur - la croix prend tout son sens et la lettre latérale est alors considérée comme une marque de pose. Dans le cas où l'on opte pour des signa pragmatiques, l'utilisation sacralisée du plateau et la piété du marbrier seraient soulignées par la croix initiale, la lettre suivante désignant un lot ou une indication de montage de la colonnette d'angle. Cette dernière opinion avait en 1983 la faveur de Jean-Pierre Sodini, dans la droite ligne des recensements effectués sur des éléments architecturaux par F. Deichmann à Ravenne ${ }^{27}$. Dans son étude d'un exemple de la basilique du Letôon de Xanthos ${ }^{28}$, il rappelait au passage les exemples d'autels cités dans les années 1950 par A.K. Orlandos ${ }^{29}$. En comparant plus récemment la mensa de Xanthos (où l'on retrouve un monogramme précédé d'une croix et suivi de la lettre de positionnement $n u$ ) aux exemples des deux sites albanais déjà cités, il admet aujourd'hui avec l'équipe des auteurs du volume Byllis $\mathrm{I}^{30}$, l'interprétation de Skënder Muçaj, l'archéologue qui a découvert les pièces bylliotes au dispositif inscrit si comparable: le monogramme figurant au revers des mensae pourrait être le nom du commanditaire, un évêque de la ville - celui de Byllis étant connu par des inscriptions sur mosaïques de datation contemporaine.

Plus qu'exclusives l'une de l'autre, considérons que ces deux catégories (commanditaire et marbrier) sont probablement complémentaires. Il faudrait d'ailleurs leur ajouter de possibles marques inscrites a posteriori, par exemple quand on a plusieurs monogrammes différents et visibles sur la table et non sous elle comme à Chios $^{31}$ (deux religieux utilisateurs de l'autel ?).

Notons pour finir que, comme à Byllis, Saranda, Sirmium ${ }^{32}$, Chypre, Xanthos, Khirbet Beit Sila ${ }^{33}$, etc., à Martinšćica qui est à l'heure actuelle le cas le plus septentrional, le signum monogrammatique est placé sous le plateau et dissimulé aux regards. Cette revendication invisible de l'œuvre, qu'elle émane du marbrier ou plutôt à notre sens du commanditaire (un évêque d'Osor ?), lui garantissait de profiter durablement de la grâce générée par toutes les synaxes eucharistiques que l'autel allait accueillir une fois mis en place dans l'église Saint-Martin.

\section{Datation du mortier provenant de l'annexe VIIla par luminescence optiquement stimulée, méthode dite « single grain » (P. Urbanová)}

Les difficultés pour établir la chronologie absolue de certaines phases de construction à Martinšćica nous ont amenés à faire appel à la datation des mortiers par luminescence optiquement stimulée, réalisées au laboratoire de Bordeaux IRAMAT-CRPAA (thèse de P. Urbanová, 2012-2015) ${ }^{34}$. Cette nouvelle méthodologie permettant de dater le mortier 
et dont la fiabilité a été récemment validée par une série d'études chronologiques sur des édifices d'âge connu ${ }^{35}$, devient aujourd'hui une méthode de travail prometteuse pour accéder à la chronologie du bâti ${ }^{36}$. Dans ces lignes sont présentés les premiers résultats de datation de l'annexe VIIIa obtenus grâce à cette approche.

\section{Principes généraux de la méthode employée}

La luminescence optiquement stimulée (OSL) est une méthode de datation paléodosimétrique ${ }^{37}$ qui exploite les effets de la radioactivité naturelle sur les minéraux de quartz ou de feldspath. Elle permet de dater le moment de leur dernière exposition à la lumière. Dater le mortier par OSL signifie dater le moment où les grains de sable du mortier ont été exposés à la lumière pendant le mélange avec la chaux, c'est-à-dire, au moment de la préparation du mortier. Ce processus de remise à zéro de grains par la lumière s'appelle le "blanchiment» et correspond, en théorie, au moment de l'édification du bâti.

De nombreux essais permettant de dater le mortier par OSL ont été entrepris dans le passé par une technique "classique» qui consiste à mesurer la luminescence de plusieurs dizaines ou centaines de grains ensemble. Cependant, les auteurs ont constaté que cette méthode d'analyse conduit dans la majorité de cas à une surestimation des âges attendus ${ }^{38}$. En effet, les mortiers contiennent souvent des grains mal blanchis qui n'ont pas été assez exposés à la lumière au moment de la fabrication du liant. En conséquence, ces grains émettent une luminescence trop élevée qui pollue la mesure.

L'innovation principale de l'approche récemment mise en pratique à Bordeaux pour les mortiers consiste à l'emploi systématique de la technique dite «single grain » (SGOSL) ${ }^{39}$. Cette méthode d'analyse permet de détecter la luminescence de chaque grain individuellement et donc de distinguer les différents degrés de blanchiment des grains dont seulement une partie porte une information chronologique sur la construction $\mathrm{du}$ bâtiment. L'utilisation de la technique SG-OSL pour dater le mortier présente dans ce contexte une avancée essentielle car celle-ci permet d'identifier par des considérations internes à la méthode des grains polluants et ainsi conduit à des résultats de datation fiables.

\section{Prélèvement pour l'OSL à l'abri de toute lumière}

Le mortier BDX 19662 de l'annexe VIIIa a été prélevé dans la partie inférieure du mur 8.104 (fig. 20). Afin d'éviter sa remise à zéro par la lumière au moment du prélèvement, l'échantillon a été pris à l'abri de toute lumière. Ceci demandait une installation imperméable à la lumière du jour, créée à l'aide de plusieurs couches de bâches (fig. 21). Une fois dans le noir, la partie superficielle du liant a été enlevée. Le mortier pour la datation SG-OSL a été prélevé par le grattage de la partie intérieure du mur qui n'a pas vu la lumière du jour depuis son édification. 
Fig. 20 - Localisation du prélèvement du mortier dans le mur 8.104 de l'annexe VIIla.

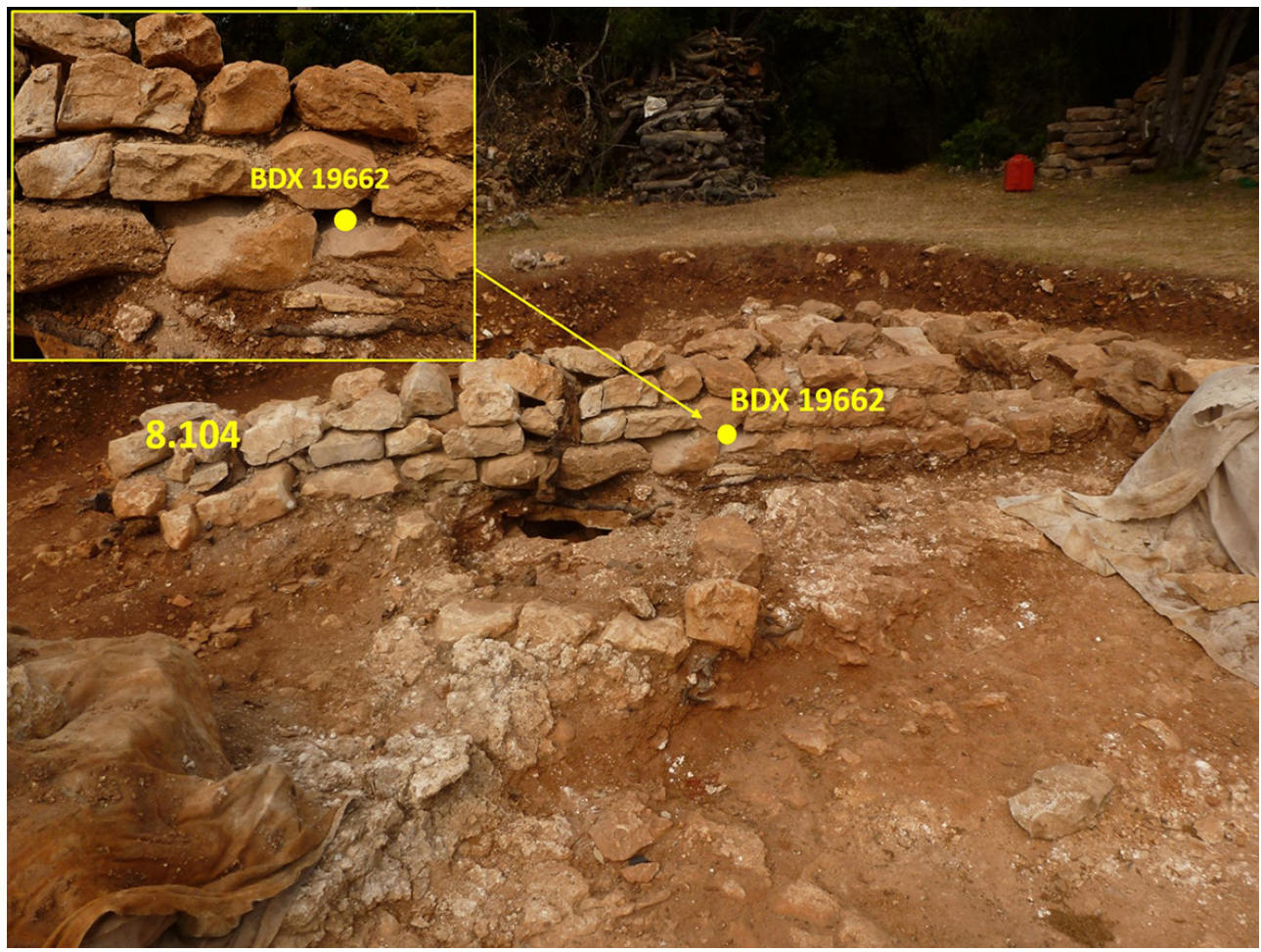

Cl. P. Urbanová

Fig. 21 - Installation permettant d'isoler de la lumière ambiante le processus de prélèvement du mortier pour la datation SG-OSL.

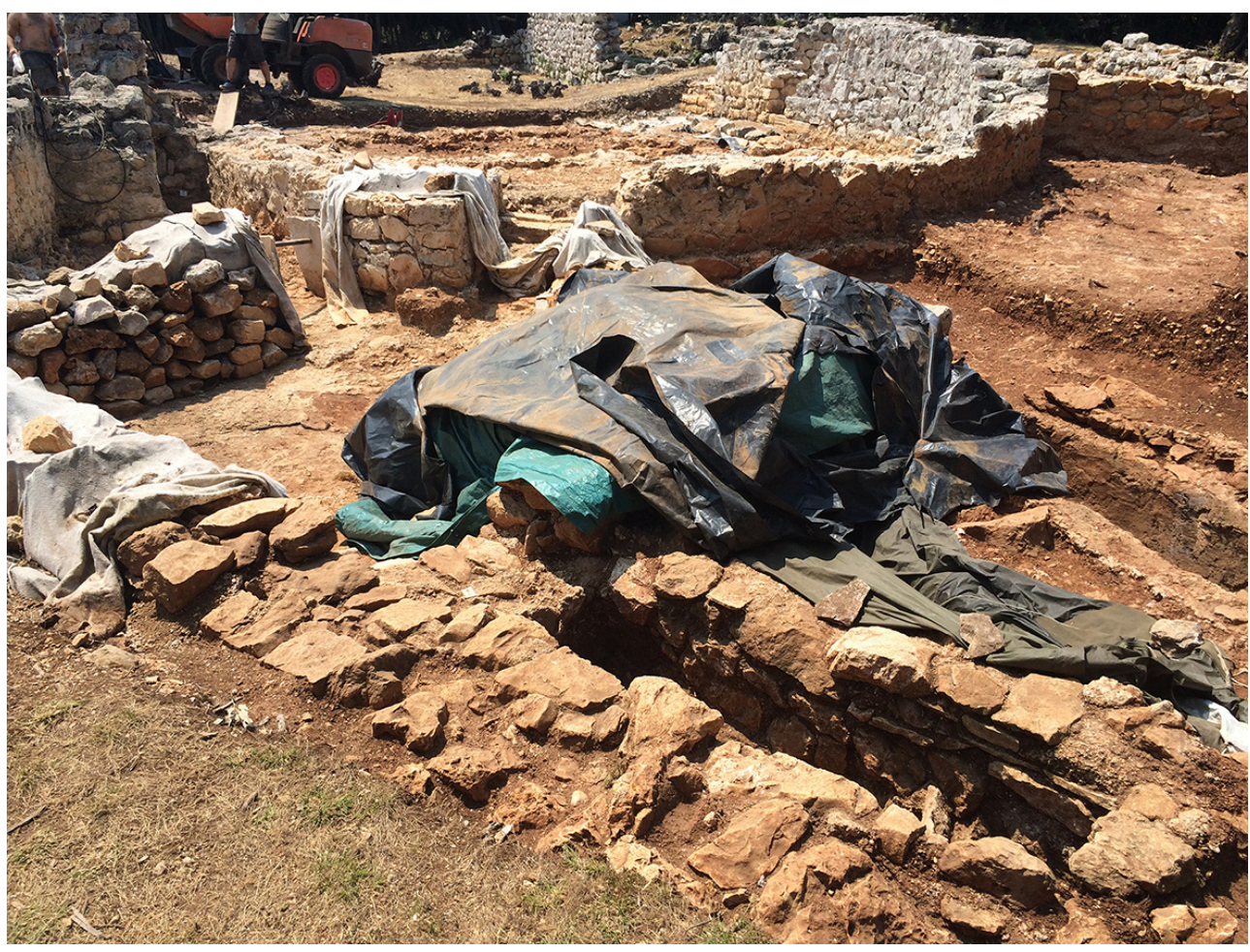

Cl. S. Bully. 


\section{Processus de datation et résultats}

La détermination de l'âge par SG-OSL consiste à calculer le rapport de la « dose archéologique » à « la dose annuelle » selon l'équation d'âge suivante :

Âge = dose archéologique/dose annuelle

La dose archéologique, exprimée en grays $(1 \mathrm{~Gy}=1 \mathrm{~J} / \mathrm{kg})$, correspond à l'énergie accumulée par les grains de quartz dans le mortier depuis sa pose dans la maçonnerie. Elle se détermine par une mesure de luminescence - la lumière - émise par les grains de quartz extraits de l'échantillon daté.

La dose annuelle, exprimée en Gy/an, représente la dose absorbée en un an par les grains de quartz dans le mortier sous l'effet de leur propre radioactivité et de celle de leur environnement. Son évaluation nécessite de conduire plusieurs types d'analyse dont la partie est réalisée in situ ${ }^{40}$.

\section{Dose archéologique globale}

3700 grains de quartz de diamètre compris entre 100 et $140 \mu \mathrm{m}$ ont été analysés un par un, par une technique de «single grain » afin de dater le mortier BDX 19662. 113 grains ont donné les signaux de luminescence exploitable. La figure 22 montre l'histogramme des doses archéologiques qui correspondent à la luminescence émise par les grains individuels. La distribution est plutôt symétrique avec les valeurs de la moyenne et de la médiane assez proches. La majorité des doses mesurées se trouve entre 0.5 et $3 \mathrm{~Gy}$. L'ensemble de ces observations indique que le mortier de l'annexe VIIIa est globalement bien blanchi. C'est-à-dire que les grains de quartz extraits de cet échantillon ont été bien remis à zéro par la lumière au moment de la préparation du mortier et portent ainsi tous l'information chronologique sur la construction de l'annexe VIIIa. On constate une présence de seulement trois grains mal blanchis avec des doses archéologiques supérieures à $3 \mathrm{~Gy}$, qui sont facilement identifiables dans la figure 22. Le risque de surestimation de l'âge lié au mauvais blanchiment du mortier ne se pose donc pas dans ce cas précis.

Étant donné le bon état de blanchiment, tous les grains, à part les 3 considérés mal blanchis, ont pu être pris en compte pour la datation. La dose archéologique globale, calculée en utilisant le Modèle d'âge central ${ }^{41}$, est égale à $1.40 \pm 0.10 \mathrm{~Gy}$. 
Fig. 22 - Histogramme des doses archéologiques correspondant à la luminescence émise par les grains individuels.

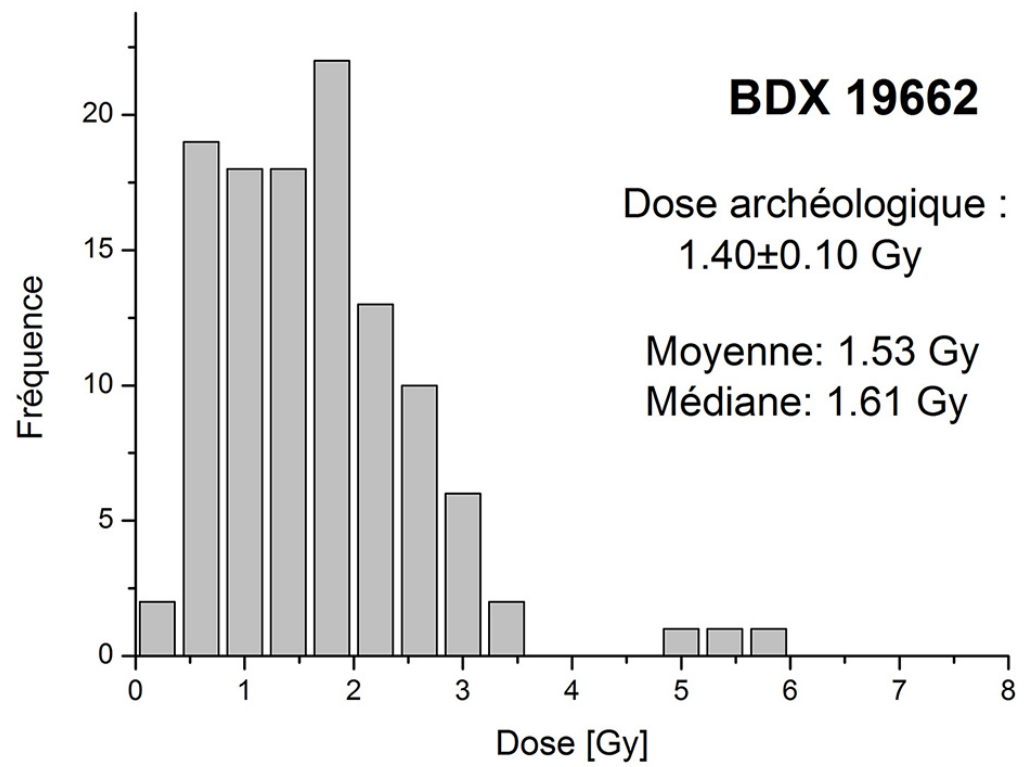

Les mesures ont été effectuées avec le lecteur TL/OSL DA20 Ris $\varnothing$ reader en appliquant le protocole SAR (A.S. Murray, A. Wintle, Luminescence dating of quartz using an improved single-aliquot regenerative dose protocol, dans Radiation Measurements, 32, 2000, p. 523-538.). Paramètres de mesure :

températures de préchauffe : $190{ }^{\circ} \mathrm{C} / 160{ }^{\circ} \mathrm{C}$, doses de régénération : $[0.54 ; 1.09 ; 1.63 ; 2.18 ; 0 ; 0.54]$ Gy, dose test : 2.72 Gy.

P. Urbanová.

L'analyse de la composition chimique (Tableau 1) montre que le mortier de l'annexe VIIIa est formé en $70 \%$ par l'oxyde de calcium ( $\mathrm{CaO}$ ). Cependant, la combinaison de ces résultats avec la cathodoluminescence (fig. 23) et l'étude pétrographique a révélé que la majorité de l'oxyde de calcium $(\mathrm{CaO})$ se trouve dans l'agrégat du mortier de nature majoritairement calcaire. Le liant est en réalité très faible en $\mathrm{CaO}$. 
Fig. 23 - Image de cathodoluminescence de la lame épaisse du mortier BDX 19662.

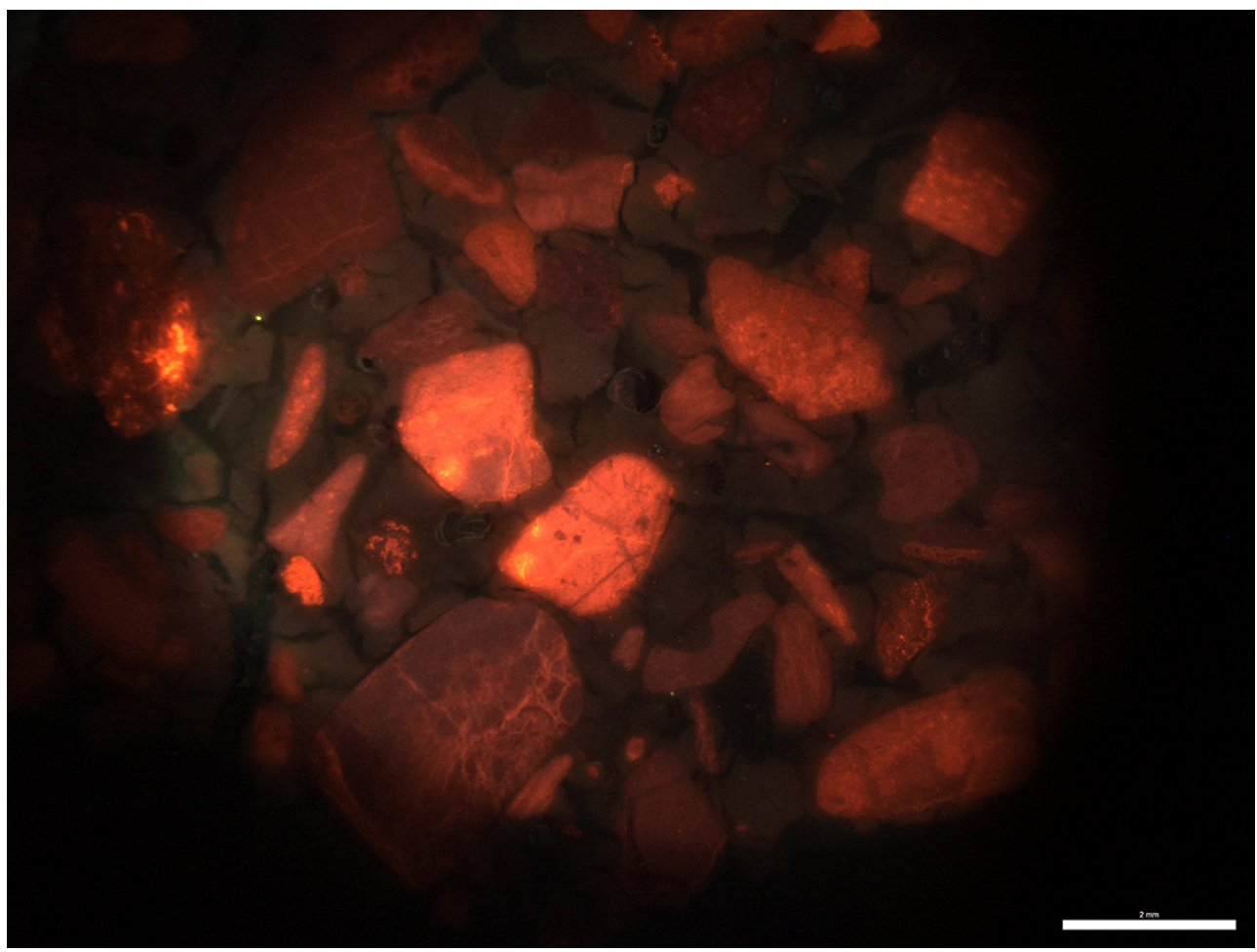

On remarque le caractère grossier du mortier très riche en fragments calcaires (en rouge).

Cl. P. Urbanová.

Tableau 1 - Composition élémentaire du mortier BDX 19662 obtenue par la microscopie électronique à balayage couplée avec le détecteur des rayons $\mathrm{X}$ pour la fraction granulométrique inférieure à $2 \mathrm{~mm}$ et à $1 \mathrm{~cm}$.

\begin{tabular}{|l|c|c|c|c|c|c|c|c|}
\hline \multirow{2}{*}{ Fraction } & \multicolumn{7}{|c|}{ Composition élémentaire exprimée en pourcentage d'oxydes } \\
\cline { 2 - 9 } & $\mathrm{NaO}$ & $\mathrm{MgO}$ & $\mathrm{Al}_{2} \mathrm{O}_{3}$ & $\mathrm{SiO}_{2}$ & $\mathrm{~K}_{2} \mathrm{O}$ & $\mathrm{CaO}$ & $\mathrm{TiO}_{2}$ & $\mathrm{Fe}_{2} \mathrm{O}_{3}$ \\
\hline$>2 \mathrm{~mm}$ & 0.3 & 1.4 & 5.9 & 18.0 & 0.5 & 70.9 & 0.3 & 2.2 \\
\hline$>1 \mathrm{~cm}$ & 0.3 & 1.5 & 5.5 & 15.3 & 0.5 & 74.4 & 0.2 & 2.9 \\
\hline
\end{tabular}

La dispersion entre les grains bien blanchis comme on peut le voir sur la figure 22 est relativement élevée, égale à $48 \pm 4 \%$. Ceci s'explique par le caractère très hétérogène de ce mortier, formé en $70 \%$ par les fragments calcaires grossiers (supérieurs à $2 \mathrm{~mm}$ ), bien visibles par cathodoluminescence (fig. 23) et par microscopie électronique à balayage (fig. 24a). Ces derniers contiennent des faibles teneurs en radioéléments, contrairement aux minéraux lourds riches en uranium, présents également dans la matrice (fig. 24b). On peut donc s'attendre à des variations microdosimétriques relativement importantes à l'échelle des grains liées à la distribution hétérogène des radioéléments dans le mortier. Par conséquent, tous les grains de quartz ne reçoivent pas exactement la même dose d'irradiation. 
Fig. 24 - Lame épaisse du mortier BDX 19662 imagée par la microscopie électronique à balayage couplée avec le détecteur des rayons $X$ sous un grossissement 60 .
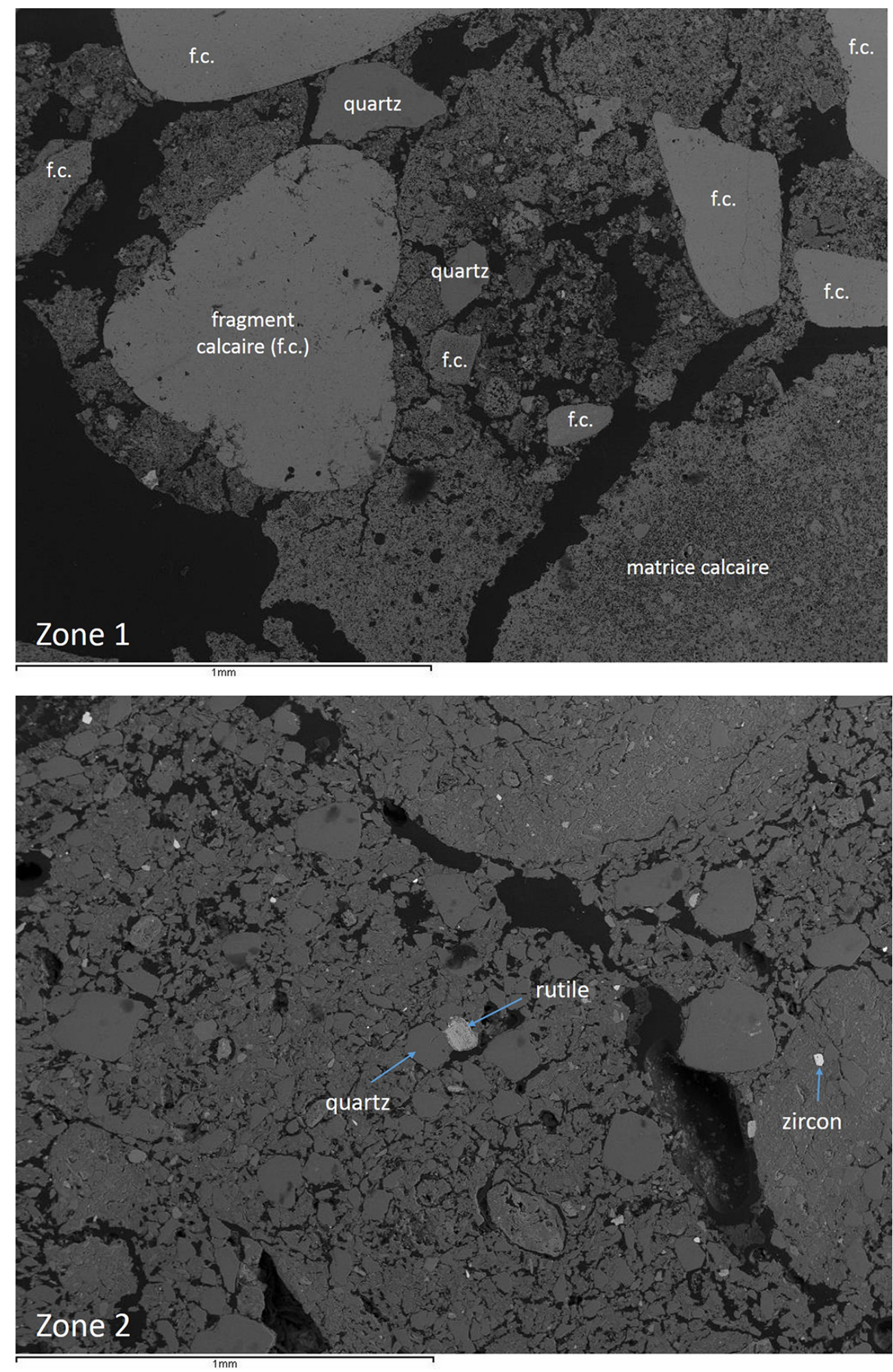

L'environnement des grains de quartz entre la zone 1 et la zone 2 est très différent. Tandis que dans la zone 1 on remarque majoritairement les fragments calcaires avec les faibles teneurs en radioéléments, dans la zone 2 on identifie plusieurs minéraux lourds (points blancs) riches en radioéléments. Ces variations expliquent en partie la dispersion dans la distribution des doses archéologiques observée parmi les grains utilisés pour la datation (fig. 22).

Cl. P. Urbanová 


\section{Dose annuelle}

La radioactivité environnementale, représentant les contributions des rayonnements gamma et cosmiques à la dose annuelle, a été déterminée par dosimétrie. Un tube métallique contenant les pastilles dosimétriques de Al203 : C sensibles aux irradiations naturelles est resté posé in situ, dans l'endroit du prélèvement, pendant une période d'un an (350 jours). La dose environnementale obtenue par dosimétrie est égale à $0.54 \pm 0.01 \mu \mathrm{Gy} /$ an ce qui indique une radioactivité très faible de l'environnement à Martinšćica.

L'annexe VIIIa a été ensevelie avant sa découverte pendant un certain temps, tandis que la mesure de radioactivité par dosimétrie a été faite après le déblayage des couches de démolition. Une partie de l'environnement du mur 8.104, initialement en élévation, était donc manquante au moment de la mesure. Cependant, le mortier a été prélevé en profondeur à $20 \mathrm{~cm}$ du mur dont l'épaisseur est égale à $56 \mathrm{~cm}$. Les rayonnements gamma ayant des effets sur l'échantillon daté proviennent donc principalement du mur qui est resté préservé. De plus, le comblement de l'annexe VIIIa a été constitué par les couches de démolition de ce même mur avec une composition radiochimique a priori similaire. Ces deux arguments nous amènent à considérer la radioactivité environnementale mesurée comme suffisamment représentative.

Pour déterminer la radioactivité du mortier, les teneurs en radioéléments ont été mesurées par spectrométrie gamma à bas bruit de fond ${ }^{42}$. Étant donné le caractère grossier du mortier BDX 19662 formé en $70 \%$ de fragments calcaires de dimensions entre $2 \mathrm{~mm}$ et $1 \mathrm{~cm}$, les teneurs en radioéléments ont été mesurées non seulement pour la fraction inférieure à $2 \mathrm{~mm}$, mais aussi pour toute la fraction inférieure à $1 \mathrm{~cm}$. Les résultats de ces deux mesures, présentés dans le tableau 2, ont été combinés selon la procédure décrite par Tribolo et al. ${ }^{43}$

Tableau 2 - Mesure des teneurs en radioéléments $K, U$ et Th déterminées par spectrométrie gamma à bas bruit de fond pour deux fractions granulométriques : une inférieure à $2 \mathrm{~mm}$ et une autre inférieure à $1 \mathrm{~cm}$.

\begin{tabular}{|c|c|c|c|c|c|c|}
\hline Échantillon & Fraction & $\begin{array}{l}\mathbf{U}(238 \mathbf{U}) \\
{[\mathbf{p p m}]}\end{array}$ & $\begin{array}{c}\left.\text { U( }{ }^{226} \mathbf{R a}\right) \\
{[p p m]}\end{array}$ & $\begin{array}{c}\mathrm{U}\left({ }^{210} \mathrm{~Pb}\right) \\
{[\mathrm{ppm}]}\end{array}$ & $\begin{array}{c}\text { Th } \\
{[\mathrm{ppm}]}\end{array}$ & $\begin{array}{c}\mathbf{K}_{\text {sp gamma }} \\
{[\%]}\end{array}$ \\
\hline BDX 19662 & $\begin{array}{l}\text { Inférieure à } \\
2 \mathrm{~mm}\end{array}$ & $2.61 \pm 0.11$ & $2.91 \pm 0.03$ & $3.69 \pm 0.41$ & $1.72 \pm 0.05$ & $0.17 \pm 0.01$ \\
\hline BDX 19662 & Inférieure à $1 \mathrm{~cm}$ & $2.71 \pm 0.10$ & $3.02 \pm 0.03$ & $3.08 \pm 0.36$ & $1.07 \pm 0.05$ & $0.11 \pm 0.01$ \\
\hline
\end{tabular}

La pureté des grains de quartz utilisés pour la datation a été contrôlée par microscopie électronique à balayage. L'analyse n'a montré aucune présence d'inclusions radioactives telles que les zircons ou les apatites à l'intérieur des grains de quartz. On considère donc la radioactivité interne aux grains comme négligeable.

L'humidité de l'échantillon au moment du prélèvement a été évaluée par la différence des poids de l'échantillon après le prélèvement et à son état sec. La teneur en eau obtenue, égale à $5 \%$, est relativement faible, ce qui s'explique par le climat sec de la zone géographique ainsi que par la grande proportion de fragments grossiers contenus dans ce mortier dont la présence limite la capacité du mortier à retenir l'eau. 
Tableau 3 - Récapitulatif des différentes contributions à la dose annuelle pour deux fractions granulométriques présentes dans le mortier. La dose annuelle prise en compte pour le calcul de l'âge représente la moyenne des doses annuelles totales déterminées pour chacune des fractions.

\begin{tabular}{|c|c|c|c|c|c|c|c|}
\hline \multirow{3}{*}{$\begin{array}{c}\text { Échantillo } \\
\mathbf{n}\end{array}$} & \multirow{3}{*}{$\begin{array}{c}\text { Fraction } \\
\text { granulométrique }\end{array}$} & \multirow{3}{*}{$\begin{array}{c}\text { Teneur } \\
\text { en eau } \\
{[\%]}\end{array}$} & \multicolumn{3}{|c|}{ Contributions à la dose annuelle } & \multirow{3}{*}{$\begin{array}{c}\text { Dose annuelle } \\
\text { totale } \\
{[\mathrm{mGy} / \mathrm{y}]}\end{array}$} & \multirow{3}{*}{$\begin{array}{c}\text { Dose annuelle } \\
\text { moyenne } \\
{[\mathrm{mGy} / \mathrm{y}]}\end{array}$} \\
\hline & & & \multicolumn{2}{|c|}{ Matrice du mortier ${ }^{\mathrm{a}}$} & \multirow{2}{*}{$\begin{array}{l}\text { Environnement }^{\mathrm{b}} \\
\begin{array}{l}\text { Dose annuelle } \mathrm{y}^{\mathrm{e}} \mathrm{et} \\
\text { cosmique [mGy/y] }\end{array}\end{array}$} & & \\
\hline & & & $\begin{array}{c}\text { Dose } \\
\text { annuelle } \alpha \\
{[\mathrm{mGy} / \mathrm{y}]}\end{array}$ & $\begin{array}{c}\text { Dose } \\
\text { annuelle } \beta \\
{[\mathrm{mGy} / \mathrm{y}]}\end{array}$ & & & \\
\hline BDX 19662 & Inf. à $2 \mathrm{~mm}$ & \multirow{2}{*}{$5 \pm 2$} & $0.05 \pm 0.02$ & $0.51 \pm 0.02$ & \multirow{2}{*}{$0.54 \pm 0.01$} & $1.10 \pm 0.04$ & \multirow{2}{*}{$1.075 \pm 0.04$} \\
\hline BDX 19662 & Inf. à $1 \mathrm{~cm}$ & & $0.05 \pm 0.02$ & $0.46 \pm 0.01$ & & $1.05 \pm 0.04$ & \\
\hline
\end{tabular}

a déterminé par spectrométrie gamma à bas bruit de fond ; b déterminé par dosimétrie in situ.

Enfin, l'ensemble des contributions de la radioactivité de l'environnement et du mortier, présentés dans le tableau 3, a été additionné afin de calculer la dose annuelle totale, égale à $1.075 \pm 0.040 \mathrm{mGy} / \mathrm{an}$. Le ratio de la dose archéologique à la dose annuelle permet de déterminer l'âge du mortier BDX 19662 égal à 1299 105 années ce qui correspond à la date $719 \pm 105$ de notre ère pour la construction de l'annexe VIIIa (tableau 4).

Tableau 4 - Récapitulatif des paramètres clés nécessaires à la datation SG-OSL du mortier de l'annexe VIIIa.

\begin{tabular}{|c|c|c|c|c|c|c|c|}
\hline Échantillon & $\begin{array}{c}\text { Nb de grains } \\
\text { analysés }\end{array}$ & $\begin{array}{c}\text { Nb de grains } \\
\text { luminescents }\end{array}$ & $\begin{array}{c}\text { Nb de grains pris } \\
\text { en compte }\end{array}$ & $\begin{array}{c}\text { Dose archéologique } \\
\text { globale [Gy] }\end{array}$ & $\begin{array}{c}\text { Dose annuelle } \\
{[\mathbf{m G y} / \mathbf{y}]}\end{array}$ & $\begin{array}{c}\text { Âge } \\
\text { [année] }\end{array}$ & Date [année] \\
\hline BDX 19962 & 3700 & 113 & 110 & $1.40 \pm 0.10$ & $1.075 \pm 0.04$ & $1299 \pm 105$ & $719 \pm 105$ \\
\hline
\end{tabular}

\section{Conclusion}

Pressentie en raison de son parti architectural à abside outrepassée, la découverte des tombes en formae dans le bras du transept sud - fonctionnant comme une annexe funéraire - semble confirmer la fonction mémorielle de la chapelle sud. En outre, la mise au jour d'un édifice à abside antérieur à cette chapelle repose la question de l'origine même de la grande église. La présence des formae - c'est-à-dire de tombes préaménagées construites simultanément - est une découverte majeure car elles constituent un indice supplémentaire pour la reconnaissance d'un milieu édilitaire (épiscopal ?) ou communautaire (monastique) ${ }^{44}$. Sous une formule architecturale différente, ces formae nous renvoient à celles découvertes dans le mausolée d'Osor à proximité de l'église abbatiale Saint-Pierre et nous interpellent sur un dispositif funéraire dont à ce jour on ne connaîtrait pas de comparaisons dans le Kvarner, et plus largement en Dalmatie.

La fonction des annexes orientales - dont on suggère désormais une datation de leur construction au début du VIII ${ }^{e}$ siècle - demeure une question ouverte, même si dans l'état actuel de notre réflexion et de la documentation nous suggérons l'hypothèse de « cellules» monastiques. 
Avec les deux annexes orientales, le sceau notarial du début du VIII ${ }^{e}$ siècle constitue l'un des témoignages les plus tardifs de l'histoire de l'occupation du site et nous interroge sur les liens entre le complexe de Martinšćica et l'administration Byzantine pour une période sur laquelle nous savons encore très peu dans la région. Ajoutons brièvement à ce dossier que des premières analyses réalisées sur des fragments de verre à vitre de l'église et de gobeleterie découverts sur le site ont révélé des compositions physico-chimiques de verre d'origine syro-palestinienne (production tardive de verres au natron) que l'on rencontre à partir du VII ${ }^{\mathrm{e}}$ siècle ${ }^{45}$.

Après trois campagnes de fouilles programmées, nous avons pris le parti de consacrer l'année 2018 exclusivement à des travaux de conservation des vestiges nécessaires à la poursuite des recherches en 2019.

\section{NOTES}

1. Nous exprimons notre gratitude envers les financeurs de ces recherches: le ministère des Affaires étrangères français, le ministère de la Culture croate, l'École française de Rome, la fondation Caritas Veritatis et la région de Primosko-Goranska. La logistique de l'opération est gérée par l'association aIPAK, Omišalj.

2. Travaux réalisés par l'entreprise Marvel, Omišalj.

3. À notre connaissance, cette méthode de datation n'avait pas encore été éprouvée sur des édifices en Croatie; les premiers résultats laissent entrevoir des perspectives stimulantes pour des constructions parfois difficilement datables par les analyses architecturales habituelles.

4. Une datation par OSL a également été menée sur l'église Saint-Pierre d'Osor (première phase de l'église) ; les résultats seront publiés ultérieurement.

5. Nous adressons nos remerciements les plus sincères à $M$. Tomaž Hartman, citoyen slovène, passionné d'histoire régionale, pour nous avoir rapporté cette découverte capitale et remis le sceau.

6. Pour un complément d'étude, V. Prigent, Byzantium and the island of Cres: new numismatic and sigillographic evidence, dans S. Bully et al. (dir.), Actes des journées d'études monastiques d'Osor de juin 2018, Saint-Pierre d'Osor (île de Cres) et le monachisme bénédictin dans l'espace adriatique, à paraître.

7. PL 112, col. 1581-1584.

8. Traduction de R. Favreau, L'écriture dans les mentalités médiévales, dans Id., Études d'épigraphie médiévale, Limoges, 1995, p. 47

9. A. Giry, Manuel de Diplomatique, Paris, 1894, p. 533.

10. M. Deloche, Étude historique et archéologique sur les anneaux sigillaires des premiers siècles du Moyen Âge, Paris, 1900, p. XIV.

11. H. Leclercq, s.v. Monogramme, dans DACL, t. XI-2, Paris, 1934, col. 2370.

12. J.-C. Cheynet, s.v. Monogramme, dans A. Vauchez (dir.), Dictionnaire encyclopédique du Moyen Âge, Paris, 1997, vol. II, p.1025. Voir aussi W. Seibt, s.v. Monogramm, dans Reallexikon zur byzantinischen Kunst, t. VI, Stuttgart, 1997, p.590-614. Voir sur ce thème les réflexions d'A. Eastmond, Monograms and the Art of Unhelpful Writing in Late Antiquity, dans B.M. Bedos-Rezak, J.F. Hamburger, Sign and design. Script as image in cross-cultural perspective (330-1600 CE), Washington DC, 2016, p. 219-236. 
13. W. Fink, Das frühbyzantinische Monogramm, JÖB, 30, 1981, p. 75-86. Voir également Id., W. Seibt, Neue Wege zur Deutung der Monogramme, dans Akten des XII. Int. Byzantinistenkongresses I/Beiheft (JÖB 31/Beiheft), Vienne, 1981, Abschnitt 1.1.

14. R. Favreau, op. cit.

15. R. Feind, Byzantinische Siegelkunde. Eine Einführung in die Sygillographie von Byzanz, 2010, en particulier chap. II.1. Monogrammsiegel, p. 128-140.

16. C'est-à-dire le type «compact» de la définition de J.-Cl. Cheynet, op. cit., appelé aussi « monogramme carré » par H. Leclercq, op. cit., col. 2380.

17. R. Feind, op. cit., p. 129-131.

18. F.W. Deichmann, Ravenna. Hauptstadt des spätantiken Abendlandes, t. II, Kommentar, vol.1, Wiesbaden-Stuttgart, 1974, p. 18 et fig. 15.

19. On notera au contraire en Occident le maintien du monogramme bloc, notamment sur les bagues sigillaires mérovingiennes, avec un passage plus tardif, carolingien, au monogramme cruciforme.

20. R. Feind, op. cit., p. 132-134.

21. Voir notamment pour sa pratique en Occident médiéval, O. Guyotjeannin, Le monogramme dans l'acte royal français (Xé-début du XIV siècle), dans P. Rück (dir.), Graphische Symbole in mittelalterlichen Urkunden. Beiträge zur diplomatischen Semiotik, Sigmaringen, 1996, p. 293-318 ; et J.B. Renault, Le monogramme dans les chartes épiscopales en Lorraine et Champagne ( $X^{e}$-XII ${ }^{e}$.), dans Annales de l'Est, 59, 2009, p. 55-90.

22. J. Bardill, Brickstamps of Constantinople, Oxford, 2004, p. 49. Voir auparavant les dates de 530-540 pour l'introduction du monogramme cruciforme en Occident et spécialement à Rome, E. Weigand, Ein bisher verkanntes Diptychon Symmachorum, dans Jahrbuch des Deutschen Archäologischen Instituts, 52, 1937, p. 130.

23. P. Chevalier, Les autels paléochrétiens des provinces d'Epirus Vetus, Epirus Nova et de Praevalis, dans Hortus Artium Medievalium, 11, 2005, p. 73-74 et fig. 11a-b-c. Le premier exemple de Byllis, celui de l'autel de la cathédrale, est en marbre de Naxos.

24. Ibid., p. 73 et fig. 10a p. 72. Dans son état initial, le socle de l'autel de la basilique $C$ de la même ville de Byllis portait ainsi auprès des encoches carrées creusées aux quatre angles pour ancrer les colonnettes les lettres A, X, Г et ГА (ibid., p. 71-72; voir également Ead., L'autel, l'ambon et le baptistère à Byllis, dans L'Illyrie méridionale et l'Épire dans l'Antiquité IV, Actes du IVecolloque international de Grenoble - 10-12 octobre 2002, Grenoble, 2004, p. 447-449).

25. D. Nikolaou, Liturgical furnishing from Early Christian basilicas of Cyprus ( $4^{\text {th }} 7^{\text {th }}$ c.), Cahiers $d u$ Centre d'études chypriotes, 43, 2013, p. 169-170 et fig.9-10 p.169. Le premier monogramme est gravé sur l'abaque lisse d'un chapiteau de colonnette de la basilique extra-muros de Kourion, un autre est incisé à l'angle de la mensa du martyrium d'Akrotiri (entre une croix et un M), le troisième est au revers de l'autel de la cathédrale de Kourion.

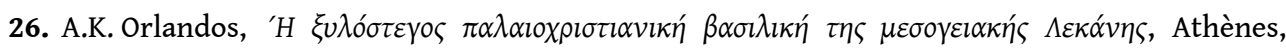
1952-1954, p. 451, et fig. 409/3, p. 450.

27. F.W. Deichmann, Ravenna. Hauptstadt des spätantiken Abendlandes, t. II, Kommentar, vol. 2, Wiesbaden-Stuttgart, 1976, p. 206-230.)

28. J.-P. Sodini, Marques de tâcheron inédites à Istanbul et en Grèce, dans X. Barral i Altet (dir.), Artistes, artisans et production artistique au Moyen Âge, Colloque international, Rennes, 1983, Paris, 1987, vol. II, Commande et Travail, p. 509 et fig. 22 p. 518. C'est l'avis auquel se range Nicolas Beaudry pour les monogrammes gravés sur les plinthes des bases cinq colonnettes de l'extraordinaire autel de Ras el Basit (N. Beaudry, Un autel et son reliquaire à Ras el Basit (Syrie du Nord), dans Hortus Artium Medievalium, 11, 2005, p.115-116, fig. 9-10); les lits d'attente des mêmes colonnettes portent une lettre de pose (deux fois $\mathrm{M}$, deux fois $\mathrm{N}$, une fois A), mais il n'y a rien de ce genre au revers de la mensa, seulement un graffiti à la pointe (A@ANA).

29. A. K. Orlandos, op. cit., en particulier sa planche fig. 409 p. 450. 
30. S. Muçaj, Byllis I : Les fortifications et les basiliques A, C et D, Athènes-Rome (sous presse).

31. Voir supra et A.K. Orlandos, op. cit.

32. Exemple inédit, à paraître prochainement nous l'espérons dans Sirmium IX.

33. S. Batz, The church of St. Theodore at Khirbet Beit Sila, dans Israel Museum Studies in Archaeology, 1, 2002, p. 48.

34. P. Urbanová, Luminescence dating of mortars by "single grain" technique and its potential building archaeology, dans Archeologia dell'Architettura, sous presse.

35. P. Urbanová et al., Multi-method dating comparison of Grimaldi castle foundations in Antibes, France, dans ArchéoSciences - Revue d'archéométrie 40, 2016, p.17-33 ; P. Urbanová, P. Guibert, A review on single grain OSL dating of mortars: a methodological study of five reference archaeological sites, dans Geochronometria, 2017, DOI: 10.1515/geochr-2015-0050.

36. P. Urbanová et al., Novel interdisciplinary approach for building archaeology: integration of mortar luminescence dating into archeological research, an example of Saint-Seurin basilica, Bordeaux, dans Journal of Archaeological Science: Reports, 20, 2018, p. 307-323, https://doi.org/10.1016/j.jasrep. 2018.04.009.

37. M.J. Aitken, An introduction to optical dating: the dating of Quaternary sediments by the use of photon-stimulated luminescence, Oxford, 1998, p. 267.

38. N. Zacharias, B. Mauz, C.T. Michael, Luminescence quartz dating of lime mortars. A first research approach, dans Radiation Protection Dosimetry, 101, 2012, p. 379-382 ; C. Goedicke, Dating historical calcite mortar by blue OSL: results from known age samples, dans Radiation Measurements, 37, 2003, p. 409-415 ; C. Goedicke, Dating mortar by optically stimulated luminescence: a feasibility study, dans Geochronometria, 38-1, 2011, p. 42-49 ; L. Panzeri, Mortar and surface dating with optically stimulated luminescence (OSL): innovative techniques for the age determination of buildings, dans Nuovo Cimento, 36 (4), 2013, p. 205-216 ; G. Stella, D. Fontana, A.M. Gueli, S.O. Troja, Historical mortars dating from OSL signals of fine grain fraction enriched in quartz, dans Geochronometria, 40-3, 2013, p. 153-164, DOI 10.2478/s13386-013-0107-8.

39. Pour la technique: G. Duller, A.S. Murray, Luminescence dating of sediments using individual mineral grains, dans Geologos, 5, 2000, p. 87-106 ; pour l'application aux mortiers : P. Urbanová, P. Guibert, A review on single grain OSL... op. cit. n. 35 .

40. Pour les détails sur l'instrumentation et sur les différentes méthodes analytiques employées, le lecteur peut se référer à l'article de P. Urbanová, P. Guibert, A review on single grain OSL... op. cit. (la version en ligne est librement accessible).

41. R.F. Galbraith et al., Optical dating of single and multiple grains of quartz from Jinmium Rock Shelter, Northern Australia: part I, experimental design and statistical models dans Archaeometry, 41 (2), 1999, p. 339-364, DOI 10.1111/j.1475-4754.1999.tb00987.x.

42. P. Guibert, M. Schvoerer, TL-dating: low background gamma spectrometry as a tool for the determination of the annual dose, dans Nuclear Tracks and Radiation Measurements, 14, 1991, p. 155-161; détails aussi dans P. Urbanová, D. Hourcade, C. Ney, P. Guibert, Sources of uncertainties in OSL dating of archaeological mortars: the case study of the Roman amphitheatre Palais-Gallien in Bordeaux, dans Radiation Measurements, 72, 2015, p. 100-110.

43. C. Tribolo et al., Across the gap: geochronological and sedimentological analyses from the late pleistocene-holocene sequence of Goda Buticha, Southeastern Ethiopia, dans PLoS ONE 12 (1): e0169418., 2017, doi:10.1371/journal.pone.0169418.

44. Sur les tombes en formae: S. Bully, Un dispositif funéraire spécifique: les formae. État de la question et nouvelles découvertes, dans Hortus Artium Medievalium, 20-2, 2014, p. 480-488.

45. Les analyses réalisées en mars 2016 et en février 2017 par Bernard Gratuze et Inès Pactat à l'UMR IRAMAT d'Orléans feront l'objet d'une publication postérieurement. 
INDEX

Index géographique : Kvarner

Mots-clés : Croatie, insularité, Antiquité tardive, haut Moyen Âge, archéologie, complexe ecclésial, monastère, formae, villa, mosaïque, sceau byzantin

institutions Université de Franche-Comté-UMR Chrono-Environnement 6249 (Besançon), UMR ARTEHIS 6298 du CNRS (Dijon-Auxerre), École française de Rome, UMR IRAMAT-CRPAA

(Bordeaux), UMR Orient et Méditerranée (Paris)

\section{AUTEURS}

\section{MORANA ČAUŠEVIĆ-BULLY}

Université Bourgogne Franche-Comté/UMR Chrono-Environnement 6249, Besançon morana.causevic-bully@univ-fcomte.fr

\section{SÉBASTIEN BULLY}

CNRS, UMR ARTEHIS 6298, Dijon-Auxerre - sebastien.bully@u-bourgogne.fr

\section{PETRA URBANOVÁ}

UMR IRAMAT-CRPAA ; université Bordeaux Montaigne - urbanpetra@seznam.cz

\section{PASCALE CHEVALIER}

Université de Clermont-Ferrand 2, UMR ARTEHIS - paskvalinac[at]gmail.com

VIVIEN PRIGENT

UMR Orient et Méditerranée (Paris) - graoute@hotmail.com 